TRANSACTIONS OF THE

AMERICAN MATHEMATICAL SOCIETY

Volume 354, Number 5, Pages 2079-2114

S 0002-9947(02)02927-6

Article electronically published on January 8, 2002

\title{
CONSTRUCTING DIVISION RINGS AS MODULE-THEORETIC DIRECT LIMITS
}

\author{
GEORGE M. BERGMAN
}

\begin{abstract}
If $R$ is an associative ring, one of several known equivalent types of data determining the structure of an arbitrary division ring $D$ generated by a homomorphic image of $R$ is a rule putting on all free $R$-modules of finite rank matroid structures (closure operators satisfying the exchange axiom) subject to certain functoriality conditions. This note gives a new description of how $D$ may be constructed from this data. (A classical precursor of this is the construction of $\mathbf{Q}$ as a field with additive group a direct limit of copies of $\mathbf{Z}$.)

The division rings of fractions of right and left Ore rings, the universal division ring of a free ideal ring, and the concept of a specialization of division rings are then interpreted in terms of this construction.
\end{abstract}

\section{INTRODUCTION}

If $f: R \rightarrow D$ is a homomorphism from a ring $R$ to a division ring $D$, then it is almost a tautology that the structure of the division subring of $D$ generated by $f(R)$ is determined by the set of rational relations satisfied by the elements of $f(R)$, i.e., the equations $r\left(f\left(x_{1}\right), \ldots, f\left(x_{n}\right)\right)=0$ that hold in $D$, where $r$ ranges over all expressions in the ring-theoretic operators and the operation ()$^{-1}$ that can be evaluated at the indicated elements.

Some interesting results on division rings were obtained using the above point of view in [1, [2]. However, an intrinsic difficulty with that approach is that the set of rational expressions $r$ that one can evaluate at a given tuple is not determined in advance. Rather, to know whether $r$ can be evaluated at $f\left(x_{1}\right), \ldots, f\left(x_{n}\right)$, i.e., whether in performing this evaluation, the operation ()$^{-1}$ is never applied to a subexpression whose value is 0 , one must know, in turn, which subexpressions equal 0 , hence which "smaller" rational relations hold.

This problem is not so serious if $R$ is commutative, since then every rational expression can be reduced to one of the form $f(a) f(b)^{-1}(a, b \in R)$, which, clearly, can be evaluated if and only if $f(b) \neq 0$, and, if evaluable, has the value 0 if and only if $f(a)=0$; thus, in that case, the structure of the division subring in question is determined by $\operatorname{Ker}(f)$. Indeed, $\operatorname{Ker}(f)$ is a prime ideal of $R$, and

Received by the editors January 24, 2000 and, in revised form, August 23, 2001.

2000 Mathematics Subject Classification. Primary 05B35, 16K40, 16S90; Secondary 16E60, 16N80, 16S50, 16U20, 18A30.

Part of this work was done in 1977 while the author was supported by the Miller Institute for Basic Research in the Sciences.

The author apologizes to workers in the field who were inconvenienced by the 23-year delay between his getting the main result of this paper, and his finding the time to prepare it for publication. 
the division ring in question is, up to isomorphism, the field of fractions of the integral domain $R / \operatorname{Ker}(f)$. For noncommutative $R$, however, $\operatorname{Ker}(f)$ does not contain enough information to determine the structure of the division ring generated by $f(R)$. For example, the free associative algebra $k\langle x, y\rangle$ in two indeterminates over a field can be embedded in two division rings $D_{1}$ and $D_{2}$ (i.e., mapped by homomorphisms both having kernel $\{0\}$ ), such that in $D_{1}$ the multiplicative group generated by $x$ and $y$ is free, while in $D_{2}$ one has $x^{-1} y=\left(y x^{-1}\right)^{2}$ [8], 77, pp. 54-55].

The big breakthrough in the study of the noncommutative case was P. M. Cohn's insight that the proper generalization of $\operatorname{Ker}(f)$ is the set of square matrices of all sizes over $R$, which, on applying $f$, yield singular matrices over $D$. He named this set the singular kernel of $f$, and characterized the sets of square matrices over a ring that can arise as singular kernels of such maps $f$, calling sets satisfying his conditions prime matrix ideals (5. Chapter 7] or 7, Chapter 4] and references at the ends of those chapters). Note that for $R$ commutative, the singular kernel of $f$ consists of those matrices whose determinants lie in $\operatorname{Ker}(f)$, so in that case, the kernel completely determines the singular kernel.

Various translations of the information given by a prime matrix ideal have been found, as surveyed in [12. The goal of this paper is to take one of these reformulations and give a new, and, I hope, enlightening construction of the division ring from that data.

Let me sketch what that formulation is. Note that if we know the singular kernel of a homomorphism $f: R \rightarrow D$, then we can also say which families of elements of a free right $R$-module $R^{n}$ map to $D$-linearly dependent families of elements of $D^{n}$. (Namely, all $m$-tuples with $m>n$, and those $m$-tuples with $m \leq n$ which, regarded as $n \times m$ matrices, have the property that all $m \times m$ minors belong to the singular kernel of $f$.) Now on any $D$-vector space, the relation of linear dependence is an example of a matroid structure (definition recalled in \$2 below), and given a homomorphism $f: R \rightarrow D$, these matroid structures on the sets $D^{n}$ induce matroid structures on the sets $R^{n}$ (namely, the above-mentioned data on which families have linear independent images), which can be shown to satisfy certain natural compatibility conditions with the $R$-module structures and with homomorphisms among $R$-modules. An axiomatization of these properties yields a concept equivalent to that of a prime matrix ideal, called in [12, §4] an algebraic matroid structure on free $R$-modules. It is this type of structure which (slightly renamed) we will take as the starting-point of our construction.

Given such a family of matroid structures on free $R$-modules, we will construct a certain directed system of (generally non-free) right $R$-modules. We will discover that the endomorphism ring of the direct limit $D_{R}$ of this system has transitivity properties which make $D_{R}$ the underlying right $R$-module of our desired division ring $D$.

In the last five sections we shall work out some classes of examples, determine what relation between two systems of matroid structures corresponds to the existence of a specialization between the corresponding division rings, and make some general observations on the type of functorial data we have used.

\section{Matroid StruCtures on Free MOdules}

The concept we shall recall next is most familiar to algebraists under names such as "abstract dependence relation". It goes back implicitly to van der Waerden 
14], who noted that results about linear dependence in vector spaces and parallel results on algebraic dependence in field extensions could be obtained by proving a small number of similar lemmas in the two cases, from which the main results (e.g., uniqueness of cardinalities of bases) could be deduced. The resulting unified axiomatic approach to linear and algebraic dependence has been used implicitly (as by van der Waerden), or explicitly (as in [6, §1.4]) in many subsequent treatments of these topics.

The same concept was introduced explicitly, and apparently independently, a few years later by H. Whitney [16], under the name "matroid", an allusion to the relation of linear dependence on the columns of a matrix, with applications to graph theory and combinatorics in mind. Since subsequent research on these structures has been largely in the latter tradition, matroid is now the most common name, and the one I will use here. Combinatorists generally restrict attention to finite matroids, a choice "justified" by the fact that a matroid structure on an infinite set is determined by the induced matroid structures on its finite subsets; but it is not natural to make such a restriction in most algebraic contexts.

An entertaining aspect of any introduction to matroids is the large number of ways of describing such a structure on a set $F$. A class of families of elements of $F$ corresponding to the linearly independent families in a vector space; a class corresponding to the minimal linearly dependent families; a class corresponding to the bases; a closure operator on $F$ satisfying conditions mimicking those of "span" in a vector space; a class of subsets corresponding to the subspaces; a class corresponding to the subspaces of codimension 1, and a function associating to every finite subset of $F$ an integer, corresponding to the dimension of the span of a subset of a vector space, all turn out, when appropriately axiomatized (with surprisingly different-looking sets of axioms) to be equivalent. We will not review that smorgasbord of elegant characterizations here (see instead [16], or [15, Chapter 1]), but shall choose one characterization and work in terms of it. (A few of the other concepts mentioned above will come up, but we will not call on the axiomatizations of matroids in terms of them.)

Definition 1. Let $F$ be a set, and $2^{F}$ its power set. Then a closure operator on $F$ means a function $\mathrm{cl}: 2^{F} \rightarrow 2^{F}$ satisfying

$$
\begin{aligned}
& (\forall X \subseteq F) \operatorname{cl}(X) \supseteq X, \\
& (\forall X, Y \subseteq F) X \subseteq Y \Rightarrow \operatorname{cl}(X) \subseteq \operatorname{cl}(Y), \\
& (\forall X \subseteq F) \operatorname{cl}(\operatorname{cl}(X))=\operatorname{cl}(X) .
\end{aligned}
$$

If $\mathrm{cl}$ is a closure operator on $F$, then a subset $X \subseteq F$ is called cl-closed if $\operatorname{cl}(X)=X$. A closure operator cl is called finitary if

$$
(\forall X \subseteq F) \operatorname{cl}(X)=\bigcup_{\text {finite } X_{0} \subseteq X} \operatorname{cl}\left(X_{0}\right) .
$$

A closure operator $\mathrm{cl}$ is said to have the exchange property if

$$
(\forall X \subseteq F, y, z \in F) z \in(\operatorname{cl}(X \cup\{y\})-\operatorname{cl}(X)) \Rightarrow y \in \operatorname{cl}(X \cup\{z\}) .
$$

$A$ matroid will mean a set $F$ given with a finitary closure operator cl having the exchange property. (Cf. [15, Theorem 1.2.4], 12, (ACXF1-4)].)

Remarks. Condition (4) implies (2), so the class of finitary closure operators could be described by (11), (3) and (4). Though we are calling a closure operator satisfying 
(4) "finitary", the term "algebraic" is more common (e.g., [12, 44), since the property is typical of constructions in algebra of the form "subobject generated by $X$ ". Condition (4) is not included in the hypotheses of [15, Theorem 1.2.4], which states that every closure operator with the exchange property arises from a matroid (as defined there, in terms of a relation of independence), because there, $F$ is assumed finite. (The interested reader can verify that that theorem from [15] goes over to not necessarily finite sets if to the closure axioms for a matroid [15. pp. 8-9, (S1)-(S4)] one adds (4) above, while to the independence axioms [15] p. 7, (I1)-(I3)] one adds the condition that a set is independent if and only if all its finite subsets are independent. Cf. [15] $\S \S 20.1-20.2]$.)

Actually, there is a condition weaker than finiteness of $F$ which will hold in the situation we are interested in, and which is sufficient to render (44) automatic in the presence of the other listed conditions:

Lemma 2. If $\mathrm{cl}$ is a closure operator on subsets of a set $F$ which has the exchange property (5), and if $F=\operatorname{cl}(Y)$ for some finite set $Y \subseteq F$, then cl is finitary (satisfies (40).

Proof. Let $Y$ be as in the statement of the lemma, and $X$ a set for which we wish to prove the equation of (4). If $n$ is the cardinality of $Y$, we may assume that among all $n$-element sets with $F$ as their closure, $Y$ includes the largest number of elements of $X$. We claim then that $\operatorname{cl}(X)=\operatorname{cl}(Y \cap X)$. For if not, let $z$ be an element of $X$ not in $\operatorname{cl}(Y \cap X)$, and let $Y_{0}$ be a minimal subset of $Y$ such that $z \in \operatorname{cl}\left(Y_{0}\right)$. Then $Y_{0}$ must contain an element $y \notin X$. Applying (5) with $Y_{0}-\{y\}$ in place of $X$, we conclude that $y \in \operatorname{cl}\left(Y_{0}-\{y\} \cup\{z\}\right)$, hence $\operatorname{cl}\left(Y_{0}-\{y\} \cup\{z\}\right) \supseteq \operatorname{cl}\left(Y_{0}\right)$, hence $\operatorname{cl}(Y-\{y\} \cup\{z\}) \supseteq \operatorname{cl}(Y)=F$. But then $Y-\{y\} \cup\{z\}$ contradicts our maximality assumption on the number of elements of $X$ in $Y$.

This proves that $\operatorname{cl}(X)=\operatorname{cl}(Y \cap X)$, so $\operatorname{cl}(X)$ is contained in the union shown in (4). The reverse inclusion is clear from (2).

Since the concept of a matroid structure on an infinite set is not natural without (4), I have made (4) part of our definition; but the above lemma shows why we will never need to call on that condition below.

Let us now relate these concepts to module theory.

Throughout this note, a ring will mean an associative ring with 1 , ring homomorphisms will respect 1 , modules will be unital, and, unless the contrary is stated, will be right modules. The zero ring will mean the unique (up to isomorphism) one-element ring; it will not be considered a division ring. We will denote by $\mathbf{N}$ the set of natural numbers $\{0,1,2, \ldots\}$.

Definition 3. Let $R$ be a ring. Then by a coherent matroidal structure on free $R$-modules we shall mean a family $\left(\mathrm{cl}_{R^{n}}\right)_{n \in \mathbf{N}}$, such that for each $n \in \mathbf{N}, \mathrm{cl}_{R^{n}}$ is a matroid structure (a finitary closure operator with exchange) on $R^{n}$, and such that for all $m, n \in \mathbf{N}$,

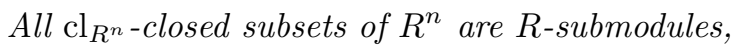

and

For every $R$-module homomorphism $h: R^{m} \rightarrow R^{n}$ and every subset $X \subseteq R^{m}$, we have $h\left(\operatorname{cl}_{R^{m}}(X)\right) \subseteq \operatorname{cl}_{R^{n}}(h(X))$. 
By the improper coherent matroidal structure on free $R$-modules, we shall mean the family of operators defined by $\operatorname{cl}_{R^{n}}(X)=R^{n}$ for every $X \subseteq R^{n}$. Any other coherent matroidal structure will be called proper.

(Note that (11) and (6) imply that, writing $\left\{e_{1}, \ldots, e_{n}\right\}$ for the standard basis of $R^{n}$, we have $R^{n}=\operatorname{cl}_{R^{n}}\left(\left\{e_{1}, \ldots, e_{n}\right\}\right)$. Thus by Lemma 2, the condition "finitary" is redundant in the above definition.)

Conditions (6) and (7) of the above definition have useful equivalent formulations:

Lemma 4. Let $\left(\mathrm{cl}_{R^{n}}\right)_{n \in \mathbf{N}}$ be a family of closure operators (not required to be matroid structures) on finitely generated free $R$-modules. Then for any nonnegative integer $n$, (6) holds if and only if

For every subset $X \subseteq R^{n}, \mathrm{cl}_{R^{n}}(X)$ contains $X R$, the submodule of $R^{n}$ generated by $X$.

Likewise, for nonnegative integers $m$ and $n$, (7) holds if and only if

For every $R$-module homomorphism $h: R^{m} \rightarrow R^{n}$, and every $\mathrm{cl}_{R^{n}-}$ closed subset $N \subseteq R^{n}$, the inverse image $h^{-1}(N)$ is a $\mathrm{cl}_{R^{m} \text {-closed }}$ subset of $R^{m}$.

Proof. Assuming (6), we see that for $X \subseteq R^{n}, \mathrm{cl}_{R^{n}}(X)$ will be an $R$-submodule of $R^{n}$ containing $X$, hence will contain $X R$, proving (8). Conversely, assuming (8), if

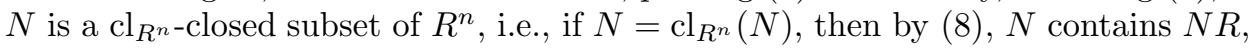
proving (6).

Assume next that (17) holds, and let $h, N$ be as in the hypothesis of (91). Applying (77) with $X=h^{-1}(N)$, then applying to the right-hand side of the resulting inclusion the observation that $h\left(h^{-1}(N)\right) \subseteq N$ and the fact that $N$ is $\mathrm{cl}_{R^{n} \text {-closed, we get }}$ $h\left(\operatorname{cl}_{R^{m}}\left(h^{-1}(N)\right)\right) \subseteq N$. This is equivalent to $\operatorname{cl}_{R^{m}}\left(h^{-1}(N)\right) \subseteq h^{-1}(N)$, i.e., $h^{-1}(N)$

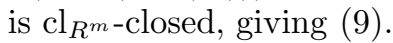

Conversely, assuming (9), let $h$ and $X$ be as in the hypothesis of (77). By (9),

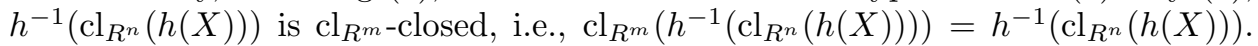
Now by (II) and (2), the left-hand side contains $\mathrm{cl}_{R^{m}}\left(h^{-1}(h(X))\right)$, which contains $\operatorname{cl}_{R^{m}}(X)$, so the above equation implies $\operatorname{cl}_{R^{m}}(X) \subseteq h^{-1}\left(\operatorname{cl}_{R^{n}}(h(X))\right)$, equivalently, $h\left(\operatorname{cl}_{R^{m}}(X)\right) \subseteq \mathrm{cl}_{R^{n}}(h(X))$, the conclusion of (7).

The fundamental examples of the above concepts are noted in

Lemma 5. Let $R$ be a ring, and for each natural number $n$ and subset $X \subseteq R^{n}$, let $\mathrm{cl}_{R^{n}}^{\bmod }(X)=X R$, the submodule of $R^{n}$ generated by $X$. Then for each $n$, the operator $\mathrm{cl}_{R^{n}}^{\bmod }$ satisfies (11)-(4) and (6), and the family of operators $\left(\mathrm{cl}_{R^{n}}^{\bmod }\right)_{n \in \mathbf{N}}$ satisfies (7). The rings $R$ such that all these operators satisfy the exchange axiom (5) (so that $\left(\mathrm{cl}_{R^{n}}^{\bmod }\right)_{n \in \mathbf{N}}$ is a coherent matroidal structure on free R-modules) are precisely the division rings and the zero ring. Of these, the ones such that this coherent matroidal structure is proper are the division rings.

Proof. (11)-(4) and (6)-(7) are familiar properties of the operator "submodule generated by", and (5) expresses a familiar property of linear algebra over division rings, and holds trivially when $R$ is the zero ring. Let us show that, conversely, (5) implies that $R$ is a division ring or the zero ring.

Assuming (5), let $r \in R-\{0\}$. The case of (5) where $n=1, X=\varnothing, y=1, z=r$ gives $1 \in \operatorname{cl}_{R}^{\bmod }(\{r\})=r R$, i.e., $r$ is right invertible. So every nonzero element of $R$ is right invertible, whence $R$ is a division ring or the zero ring. Clearly, only the zero ring gives the improper coherent matroidal structure. 
I claim now that, more generally, given any homomorphism $f: R \rightarrow D$ from a ring to a division ring, one can get a proper coherent matroidal structure $\left(\mathrm{cl}_{R^{n}}\right)_{n \in \mathbf{N}}$ on free $R$-modules by defining $\operatorname{cl}_{R^{n}}(X)$ to consist of all elements of $R^{n}$ whose images in the $D$-vector-space $D^{n}$ lie in the subspace spanned by the image of $X$. The development of this result sketched below is a couple of pages long because I have formulated each component step in what I felt was its natural generality. The reader may choose to read it, or to skim it, or to think through the result for him or herself and skip to the next section, where we begin the very nontrivial development of the converse result.

The use of the letter $F$ for the underlying set of a matroid in Definition 1 and in similar statements in this note is meant to suggest "free module", although when that letter is used, we do not generally assume a free module structure given. In the next lemma, " $V$ " is likewise meant to suggest "vector space", though again we do not assume such a structure given.

Lemma 6. Let $F$ and $V$ be sets and $s: F \rightarrow V$ a set map, and let $\mathrm{cl}_{V}$ be a map $2^{V} \rightarrow 2^{V}$. Let us define $\mathrm{cl}_{F}: 2^{F} \rightarrow 2^{F}$ by setting, for all $X \subseteq F$,

$$
\operatorname{cl}_{F}(X)=s^{-1}\left(\operatorname{cl}_{V}(s(X))\right) .
$$

Then if $\mathrm{cl}_{V}$ satisfies (1), respectively (2), respectively (4), respectively (5), then $\mathrm{cl}_{F}$ also satisfies that condition. Further, assuming $\mathrm{cl}_{V}$ satisfies (11) and (2), if it satisfies (3), then so does $\mathrm{cl}_{F}$.

Suppose next that we are given rings $R$ and $S$, an $R$-module structure on the set $F$, and an $S$-module structure on the set $V$, such that the inverse image under $s$ of every $S$-submodule of $V$ is an $R$-submodule of $F$, and such that, as in (6) , for every $Y \subseteq V$ the set $\operatorname{cl}_{V}(Y)$ is an $S$-submodule of $V$. Then $\operatorname{cl}_{F}$ likewise satisfies (6), i.e, for every $X \subseteq F$ the set $\operatorname{cl}_{F}(X)$ is an $R$-submodule of $F$.

Finally, suppose we have four sets (not assumed to have module-structures), a commuting square of maps

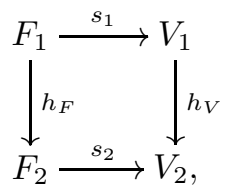

and two operators $\mathrm{cl}_{V_{i}}: 2^{V_{i}} \rightarrow 2^{V_{i}}(i=1,2)$ (not necessarily satisfying any of (11) -(5) ), and suppose we denote by $\mathrm{cl}_{F_{i}}: 2^{F_{i}} \rightarrow 2^{F_{i}}(i=1,2)$ the operators induced by $\mathrm{cl}_{V_{1}}$ and $\mathrm{cl}_{V_{2}}$ as in (10), via the maps $s_{1}$ and $s_{2}$ respectively. Then if the $\mathrm{cl}_{V_{i}}$ satisfy the analog of (7) with respect to the map $h_{V}$, namely

$$
\left(\forall Y \subseteq V_{1}\right) h_{V}\left(\operatorname{cl}_{V_{1}}(Y)\right) \subseteq \operatorname{cl}_{V_{2}}\left(h_{V}(Y)\right),
$$

then the $\mathrm{cl}_{F_{i}}$ satisfy the corresponding condition with respect to $h_{F}$,

$$
\left(\forall X \subseteq F_{1}\right) h_{F}\left(\operatorname{cl}_{F_{1}}(X)\right) \subseteq \operatorname{cl}_{F_{2}}\left(h_{F}(X)\right) .
$$

Proof. The verifications are straightforward; I will merely sketch the slightly more complicated proof of the assertion about (3) at the end of the first paragraph of the lemma.

Since in that case we are assuming that $\mathrm{cl}_{V}$, and hence $\mathrm{cl}_{F}$, satisfies (11), we need only prove the direction " $\subseteq$ " of the desired equality. If we expand $\operatorname{cl}_{F}\left(\mathrm{cl}_{F}(X)\right)$ using the definition (10), the result contains two applications of $\mathrm{cl}_{V}$, separated by the operation of taking the inverse image under $s$ and then applying $s$ to the result. 
The latter pair of operations can at worst decrease the set in question, hence, since we are assuming (2), we see that if $\mathrm{cl}_{V}$ satisfies the desired inclusion condition, so does $\mathrm{cl}_{F}$.

The consequences of the above lemma that we will use are summarized as

Corollary 7. Suppose $f: R \rightarrow S$ is a ring homomorphism, and $\left(\operatorname{cl}_{S^{n}}\right)_{n \in \mathbf{N}}$ is a coherent matroidal structure on free $S$-modules. For each nonnegative integer $n$, let $\bar{f}: R^{n} \rightarrow S^{n}$ denote the operation of applying $f$ entrywise to $n$-tuples of elements of $R$. Then the system of operators $\left(\mathrm{cl}_{R^{n}}\right)_{n \in \mathbf{N}}$ defined by setting, for all $X \subseteq R^{n}$,

$$
\operatorname{cl}_{R^{n}}(X)=\bar{f}^{-1}\left(\operatorname{cl}_{S^{n}}(\bar{f}(X))\right)
$$

is a coherent matroidal structure on free $R$-modules. If $\left(\mathrm{cl}_{S^{n}}\right)_{n \in \mathbf{N}}$ is not the improper coherent matroidal structure on free $S$-modules, then $\left(\mathrm{cl}_{R^{n}}\right)_{n \in \mathbf{N}}$ is likewise not the improper coherent matroidal structure on free $R$-modules.

If $\left(\mathrm{cl}_{S^{n}}\right)_{n \in \mathbf{N}}$ is merely assumed to satisfy (1) - (4), (6) and (7) (but not necessarily (5)), then $\left(\mathrm{cl}_{R^{n}}\right)_{n \in \mathbf{N}}$ will likewise satisfy these six conditions.

Proof. Assuming $\left(\mathrm{cl}_{S^{n}}\right)_{n \in \mathbf{N}}$ is a coherent matroidal structure, the preceding lemma shows immediately that the operations $\mathrm{cl}_{R^{n}}$ satisfy (11)-(5). (6) also follows from that lemma given the observation that the inverse image under $\bar{f}$ of an $S$-submodule of $S^{n}$ is an $R$-submodule of $R^{n}$. We similarly get (7) from that lemma and the observation that any $R$-module homomorphism $h: R^{m} \rightarrow R^{n}$ forms a commuting square with the $\bar{f}$ 's and some $S$-module homomorphism $S^{m} \rightarrow S^{n}$. (The latter can be obtained by applying the functor $-\otimes_{R} S$ to the homomorphism $h$.) We get the final sentence of the lemma by leaving (5) out of this argument.

To get the last assertion of the first paragraph of the lemma, suppose $\left(\mathrm{cl}_{S^{n}}\right)_{n \in \mathbf{N}}$ is a proper coherent matroidal structure on free $S$-modules. Then for some $n$, $\mathrm{cl}_{S^{n}}(\varnothing)$ is a proper submodule of $S^{n}$, hence it fails to contain some member $e_{i}$ of the canonical basis $e_{1}, \ldots, e_{n}$ of that module. By construction, $\mathrm{cl}_{R^{n}}(\varnothing)$ will fail to contain the corresponding basis element $e_{i} \in R^{n}$, so $\left(\mathrm{cl}_{R^{n}}\right)_{n \in \mathbf{N}}$ is also proper.

Combining with Lemma 5, we get

Corollary 8. If $f: R \rightarrow S$ is a ring homomorphism, then the system of operators $\left(\mathrm{cl}_{R^{n}}\right)_{n \in \mathbf{N}}$ defined by setting, for all $X \subseteq R^{n}$,

$$
\operatorname{cl}_{R^{n}}(X)=\bar{f}^{-1}\left(\operatorname{cl}_{S^{n}}^{\bmod }(\bar{f}(X))\right)
$$

satisfies (1) -(4), (6) and (7). If $S$ is in fact a division ring $D$, then $\left(\mathrm{cl}_{R^{n}}\right)_{n \in \mathbf{N}}$ also satisfies (5) and is proper; i.e., it constitutes a proper coherent matroidal structure on free $R$-modules.

Note that for $f: R \rightarrow D$ as in the final assertion above, the closure operator $\mathrm{cl}_{R^{n}}$ takes each subset $X \subseteq R^{n}$ to the inverse image in $R^{n}$ of the vector subspace $\bar{f}(X) D \subseteq D^{n}$; so we have established the claim made following Lemma 5 . 
The reader can easily check the following two examples of our construction, which we will refer to several times below:

Let $R=\mathbf{Z}$, and let us write $\left(\operatorname{cl}_{\mathbf{Z}^{n}}\right)_{n \in \mathbf{N}}$ for the system of closure operators induced by the unique homomorphism $\mathbf{Z} \rightarrow \mathbf{Q}$, and $\left(\mathrm{cl}_{\mathbf{Z}^{n}}^{\prime}\right)_{n \in \mathbf{N}}$ for the system induced by the unique homomorphism $\mathbf{Z} \rightarrow \mathbf{Z} / p \mathbf{Z}$ for some prime $p$. Then $\operatorname{cl}_{\mathbf{Z}^{n}}(X)$ can be described as the set of those elements $x \in \mathbf{Z}^{n}$ such that for some nonzero integer $n, n x$ lies in the additive subgroup generated by $X$, while $\mathrm{cl}_{\mathbf{Z}^{n}}^{\prime}(X)$ is the additive subgroup of $\mathbf{Z}^{n}$ generated by $X$ and $p \mathbf{Z}^{n}$.

Having verified that a homomorphism of $R$ into a division ring induces a proper coherent matroidal structure on free $R$-modules, we shall show in the next two sections how, inversely, one can construct from any such coherent matroidal structure a division ring $D$ and a homomorphism $f: R \rightarrow D$.

\section{EXTENDING CL TO NON-FREE MODULES}

We will begin with some arguments which do not require all the conditions defining a coherent matroidal structure. Hence,

Throughout this section, we shall assume that $R$ is a ring, and $\left(\mathrm{cl}_{R^{n}}\right)_{n \in \mathbf{N}}$ a family of closure operators (operators satisfying (1)-

(3) ) on finitely generated free $R$-modules, which also satisfy ([6) (closed subsets are submodules; equivalently, (8)), and (77) (functoriality, equivalently, (9) ).

The next lemma shows how to extend these operators to operators $\mathrm{cl}_{M}$ on general finitely generated $R$-modules $M$. Note that while in Lemma 6] we "pulled closure operators back" from $S$-modules to $R$-modules, here we will "push them forward" from free $R$-modules to general $R$-modules.

Lemma 9. For each finitely generated $R$-module $M$, let us define an operator $\mathrm{cl}_{M}$ on subsets of $M$ by choosing a surjective module homomorphism $g: R^{n} \rightarrow M$, and for every $X \subseteq M$ setting

$$
\operatorname{cl}_{M}(X)=g\left(\operatorname{cl}_{R^{n}}\left(g^{-1}(X \cup\{0\})\right)\right) .
$$

Then these operators are independent of our choice of $n$ and $g$, agree when $M$ is a free module $R^{n}$ with the original operators $\mathrm{cl}_{R^{n}}$, and again satisfy (11)-(3), the analog of (6) with $M$ in place of $R^{n}$, and the analog of (77):

If $h: M \rightarrow M^{\prime}$ is a homomorphism of finitely generated $R$-modules, and $X$ a subset of $M$, then $h\left(\operatorname{cl}_{M}(X)\right) \subseteq \operatorname{cl}_{M^{\prime}}(h(X))$,

as well as the following generalization of the condition by which we defined them:

If $h: M \rightarrow M^{\prime}$ is a surjective homomorphism of finitely generated $R$-modules, and $X$ is a subset of $M^{\prime}$, then $\operatorname{cl}_{M^{\prime}}(X)=$ $h\left(\operatorname{cl}_{M}\left(h^{-1}(X \cup\{0\})\right)\right)$.

If the given operators $\mathrm{cl}_{R^{n}}$ also satisfy (4), respectively (5), then so do the new operators $\mathrm{cl}_{M}$.

Proof. The occurrence of " $\cup\{0\}$ " in (15) is an artifice to avoid anomalous behavior when $X=\varnothing$ if $\operatorname{cl}_{R^{n}}(\varnothing)$ fails to contain $\operatorname{cl}_{R^{n}}(\operatorname{Ker}(g))$. We shall in fact prove most of our assertions for the simpler construction $X \mapsto g\left(\operatorname{cl}_{R^{n}}\left(g^{-1}(X)\right)\right)$, from which the results for $g\left(\operatorname{cl}_{R^{n}}\left(g^{-1}(X \cup\{0\})\right)\right)$ will follow. The " $\cup\{0\}$ " will be crucial only in 
dealing with conditions (3) and (5), and then only to insure that all sets to which $g \circ \mathrm{cl}_{R^{n}} \circ g^{-1}$ is applied are nonempty.

Let us first prove that $g\left(\mathrm{cl}_{R^{n}}\left(g^{-1}(X)\right)\right)$ is independent of the choice of $n$ and $g$. If $g: R^{n} \rightarrow M$ and $g^{\prime}: R^{n^{\prime}} \rightarrow M$ are two surjective homomorphisms, then since $R^{n}$ and $R^{n^{\prime}}$ are projective modules, we can find homomorphisms $h: R^{n^{\prime}} \rightarrow R^{n}$, $h^{\prime}: R^{n} \rightarrow R^{n^{\prime}}$ such that $g^{\prime} h^{\prime}=g, g h=g^{\prime}$. We see that $h$ will carry $g^{\prime-1}(X)$ into $g^{-1}(X)$, hence by (7) and (2), $h\left(\operatorname{cl}_{R^{n^{\prime}}}\left(g^{\prime-1}(X)\right)\right) \subseteq \operatorname{cl}_{R^{n}}\left(g^{-1}(X)\right)$; so applying $g$ to both sides, we get $g^{\prime}\left(\mathrm{cl}_{R^{n^{\prime}}}\left(g^{\prime-1}(X)\right)\right) \subseteq g\left(\mathrm{cl}_{R^{n}}\left(g^{-1}(X)\right)\right)$. The reverse inclusion holds for the same reason, so these two sets are equal, as claimed. In particular, we have this equality with $X \cup\{0\}$ in place of $X$.

When $M=R^{n}$ we can take for $g$ the identity map of that module, and conclude that in this case our construction returns the original operator $\mathrm{cl}_{R^{n}}$. (In view of (6), $0 \in \operatorname{cl}_{R^{n}}(X)$ for all $X$, so the $\cup\{0\}$ of our definition does not disturb this equality.)

To see (17), we evaluate $\operatorname{cl}_{M}\left(h^{-1}(X \cup\{0\})\right)$ using any surjective homomorphism $g: R^{n} \rightarrow M$, and $\mathrm{cl}_{M^{\prime}}(X)$ using the composite map $h g$, and see that the latter submodule will be the image of the former under $h$.

That conditions (1), (2), (6) and (7) are inherited by the $\mathrm{cl}_{M}$ with the obvious formal adjustments (e.g., (7) as (16) ), and that (4) is also inherited if it was satisfied by the original operators, is straightforward to check. In each case, one may check the condition for the operation $X \mapsto g\left(\mathrm{cl}_{R^{n}}\left(g^{-1}(X)\right)\right)$, and then apply it to $X \cup\{0\}$.

It remains to prove that $\mathrm{cl}_{M}$ satisfies (3), and satisfies (5) if that is satisfied by $\mathrm{cl}_{R^{n}}$. Let us first note

$$
\begin{aligned}
& \text { If } g: R^{n} \rightarrow M \text { is surjective homomorphism and } X \text { is a nonempty } \\
& \text { subset of } M \text {, then } g^{-1}\left(g\left(\operatorname{cl}_{R^{n}}\left(g^{-1}(X)\right)\right)\right)=\operatorname{cl}_{R^{n}}\left(g^{-1}(X)\right) .
\end{aligned}
$$

Indeed, the right-hand side is a submodule of $R^{n}$ which contains the inverse image under $g$ of a member of $M$, i.e., a coset of $\operatorname{Ker}(g)$, hence it contains $\operatorname{Ker}(g)$. Hence it is closed under adding $\operatorname{Ker}(g)$, i.e., under the operation $g^{-1} g$, as claimed.

Clearly, to show that the $\mathrm{cl}_{M}$ satisfy (3), it suffices to show that for any surjective homomorphism $g: R^{n} \rightarrow M$, the operator $g \circ \mathrm{cl}_{R^{n}} \circ g^{-1}$ is idempotent when applied to nonempty subsets $X \subseteq M$. Now when we square that operator and apply it to $X$, the result contains the composite $g^{-1} g$, applied to $\mathrm{cl}_{R^{n}}\left(g^{-1}(X)\right)$. Hence by (18), that $g^{-1} g$ can be dropped, leaving a composite containing the sequence $\mathrm{cl}_{R^{n}} \mathrm{cl}_{R^{n}}$. Since $\mathrm{cl}_{R^{n}}$ satisfies (3) we may simplify this sequence to $\mathrm{cl}_{R^{n}}$, obtaining the desired equality.

Finally, suppose $\mathrm{cl}_{R^{n}}$ satisfies (5). Given $X \subseteq M, y, z \in M$ satisfying

$$
z \in\left(\operatorname{cl}_{M}(X \cup\{y\})-\operatorname{cl}_{M}(X)\right),
$$

let $y_{0}, z_{0} \in R^{n}$ be arbitrary preimages of $y$ and $z$. Because of the " $\cup\{0\}$ " in our definition of $\mathrm{cl}_{M}$, we can assume without loss of generality that $0 \in X$ and so replace $\mathrm{cl}_{M}$ in (19) with $g \circ \mathrm{cl}_{R^{n}} \circ g^{-1}$. If to the resulting assertion we apply $g^{-1}$, and then simplify using (18), we get $z_{0} \in\left(\operatorname{cl}_{R^{n}}\left(g^{-1}(X) \cup\left\{y_{0}\right\}\right)-\operatorname{cl}_{R^{n}}\left(g^{-1}(X)\right)\right)$. Applying the assumption that $\mathrm{cl}_{R^{n}}$ satisfies (5) and, taking images in $M$, we get the conclusion of (5) for $\mathrm{cl}_{M}$.

(To see that the " $\cup\{0\} "$ in (15) is needed for (3) and (5) to hold, let $R=\mathbf{Z}$, so that $R$-modules are abelian groups, and let $\left(\mathrm{cl}_{\mathbf{Z}^{n}}\right)_{n \in \mathbf{N}}$ be the coherent matroidal structure determined by the homomorphism $\mathbf{Z} \rightarrow \mathbf{Q}$, described in (13). We find that the operators $\mathrm{cl}_{M}$ for general finitely generated abelian groups $M$ have the same description as was given there for free abelian groups $\mathbf{Z}^{n}$. Now let $g$ be a 
homomorphism from $\mathbf{Z}^{n}$ onto an abelian group $M$. We find that $g\left(\operatorname{cl}_{\mathbf{Z}^{n}}\left(g^{-1}(\varnothing)\right)\right)=$ $\{0\}$, while $g\left(\operatorname{cl}_{\mathbf{Z}^{n}}\left(g^{-1}(\{0\})\right)\right)$ is the torsion subgroup of $M$, so if $M$ has torsion, the operator $g \circ \mathrm{cl}_{R^{n}} \circ g^{-1}$ is not idempotent when applied to $\varnothing$. Likewise, letting $X=\varnothing$, letting $z$ be any nonzero torsion element of $M$, and letting $y$ be any non-torsion element, we have a counterexample to (5).)

(Note, incidentally, that we would not have needed this $\cup\{0\}$ if we had restricted our closure operators to act on submodules rather than arbitrary subsets, and that these restricted operators would have given the same information as the operators we have defined. But I am using a formulation in terms of operators on arbitrary subsets to keep the connection with the standard concept of matroid straightforward.)

Below, we shall understand $\mathrm{cl}_{M}$ to be defined for every finitely generated $R$ module $M$ via (15), and shall call a submodule of a finitely generated $R$-module $M$ "cl-closed" if it is closed under $\mathrm{cl}_{M}$. As in the preceding section, the analog of (6) for these extended operators gives us the analog of (8), i.e., that any closed subset of $M$ is a submodule, while the analog of (7), i.e., (16), gives the analog of (9), namely

The inverse image of a cl-closed submodule under a homomorphism of finitely generated $R$-modules is cl-closed.

Corollary 10. If $M$ is a finitely generated $R$-module and $N$ a cl-closed submodule, then in $M / N$, the zero submodule is cl-closed.

In particular, for any finitely generated $R$-module $M$, if we write $T(M)=$ $\operatorname{cl}_{M}(\{0\})$, then $T(M / T(M))=\{0\}$.

Proof. To get the first statement, apply (17) with $h$ the quotient map $M \rightarrow M / N$ and $X=\{0\} \subseteq M / N$. The second statement follows.

Remarks. The above corollary, together with the observation that for any morphism $h: M \rightarrow M^{\prime}$ of finitely generated $R$-modules, (16) gives $h(T(M)) \subseteq T\left(M^{\prime}\right)$, says that $T$ is a radical on the category of finitely generated $R$-modules [10 p. 2]. In fact, to give a family of closure operators $\left(\mathrm{cl}_{R^{n}}\right)_{n \in \mathbf{N}}$ as in (14) is equivalent to giving such a radical $T$, via the above construction. (Radicals are usually studied on the category of all modules over a ring $R$. A radical on finitely generated $R$-modules has a canonical extension to a radical on all $R$-modules, but we will not need this.)

The radicals most often considered in the literature are those corresponding to torsion theories. These are characterized by the additional property that for any submodule $A \subseteq M, T(A)=A \cap T(M)$; a larger class, defining what are called pretorsion theories, is characterized by the idempotence condition $T(T(M))=T(M)$ [10, p. 3]. But the cases we will be interested in will not in general have either of these properties. For instance, if $\left(\operatorname{cl}_{\mathbf{Z}^{n}}\right)_{n \in \mathbf{N}}$ is the coherent matroidal structure on $\mathbf{Z}$-modules induced by the homomorphism $\mathbf{Z} \rightarrow \mathbf{Z} / p \mathbf{Z}$ for $p$ a prime (see (13) above), we find that for all $M, T(M)=p M$, which is not an idempotent operator. We will not, except in an appendix, 凤10 below, use the notation $T(M)$ or any results from the literature on radicals; but let us borrow from that subject a name for the concept we will be concerned with next.

Definition 11. A finitely generated R-module $M$ will be called cl-semisimple if $\mathrm{cl}_{M}(\{0\})=\{0\}$.

It is easy to verify 
Lemma 12. The category of cl-semisimple R-modules (i.e., the full subcategory of the category of all $R$-modules having the cl-semisimple modules as its objects) has colimits of all diagrams with finitely many objects; in particular, it has pushouts. Such colimits can be constructed by forming the colimit of the given modules as $R$-modules, and then applying the construction $M \mapsto M / \mathrm{cl}_{M}(\{0\})$.

(The above lemma is stated only for diagrams with finitely many objects to guarantee that the colimit as $R$-modules is finitely generated. We could avoid this restriction if we extended our radical to the category of all $R$-modules; however, pushouts are the only case we will need.)

The term "semisimple" in torsion theory harks back, I believe, to the study of the Jacobson radical, where a semisimple ring or module is a subdirect product of simple objects. In the abstract theory of radicals, there is no such relationship between "semisimple" and "simple"; but in this note, a concept of "simple" object will also be of importance:

Lemma 13. Let $M$ be a nonzero cl-semisimple finitely generated $R$-module. Then the following conditions are equivalent:

(i) The only cl-closed submodules of $M$ are $\{0\}$ and $M$.

(ii) Every nonzero homomorphism from $M$ to a cl-semisimple $R$-module is oneto-one.

(iii) Any two homomorphisms from $M$ to a cl-semisimple $R$-module which agree on a nonzero element are equal.

Proof. (i) $\Rightarrow$ (ii) because by (20), the kernel of a homomorphism to a cl-semisimple module is cl-closed. The converse is obtained by applying (ii) to the canonical map $M \rightarrow M / N$ where $N$ is any cl-closed submodule of $M$. The equivalence of (ii) and (iii) is seen by looking at differences of homomorphisms.

Definition 14. A finitely generated $R$-module $M$ satisfying the equivalent conditions of Lemma 13 will be called cl-simple.

$A$ pointed cl-simple module will mean a pair $(M, p)$ where $M$ is a cl-simple module, and $p$ a nonzero element of $M$. For pointed cl-simple modules $(M, p)$ and $\left(M^{\prime}, p^{\prime}\right)$, we shall write $(M, p) \leq\left(M^{\prime}, p^{\prime}\right)$ if there exists a homomorphism $M \rightarrow M^{\prime}$ which takes $p$ to $p^{\prime}$. (Note that if such a homomorphism exists, it is unique by point (iii), and one-to-one by point (ii) of the preceding lemma.)

By the partially ordered set of pointed cl-simple modules we shall mean a set consisting of one representative of each isomorphism class of pointed cl-simple modules, partially ordered by the restriction of the above preordering.

Note that although the finitely generated $R$-modules form a proper class, not a set, there is a set containing at least one representative of each isomorphism class of such modules, namely the set of factor-modules $R^{n} / N$. Within this set we can take a subset consisting of one representative of each isomorphism class of of clsimple modules, and we can then form a set containing one representative of each isomorphism class of "pointings" of such modules. Hence the last paragraph of the above definition makes sense.

(If along with the conditions of (14) one assumes (4), then one can deduce by Zorn's Lemma that every nonzero cl-semisimple $R$-module, by definition finitely generated, has a maximal proper cl-closed submodule, and hence has a cl-simple homomorphic image. But we do not need this observation, since we shall later get a stronger result assuming (5).) 


\section{The Construction of $D$}

We now pass to the full hypothesis we will want.

In this section, $R$ will be a ring, and $\left(\mathrm{cl}_{R^{n}}\right)_{n \in \mathbf{N}}$ a proper coherent matroidal structure on free $R$-modules (Definition 3). As in the preceding section, we shall understand this family of operators to be extended by Lemma 9 to operators $\mathrm{cl}_{M}$ on arbitrary finitely generated $R$-modules $M$, which by the last sentence of that lemma will now be matroid structures.

The exchange axiom has the consequence,

Lemma 15. Let $M$ be a nonzero cl-semisimple module, and y any nonzero element of $M$. Then $M$ is cl-simple if (and only if) $\operatorname{cl}_{M}(\{y\})=M$.

Proof. Suppose $\operatorname{cl}_{M}(\{y\})=M$, and $N \subseteq M$ is a nonzero cl-closed submodule. Then applying (5) with $X=\varnothing$ and $z$ any nonzero element of $N$, we conclude that $y \in \operatorname{cl}_{M}(\{z\})$. Hence $M=\operatorname{cl}_{M}(\{y\}) \subseteq \operatorname{cl}_{M}(\{z\}) \subseteq N$, proving cl-simplicity. The converse is immediate, without the exchange axiom.

Lemma 16. The pair $\left(R / \mathrm{cl}_{R}(\{0\}),[1]\right)$ (where [1] denotes the image of $1 \in R$ in $\left.R / \mathrm{cl}_{R}(\{0\})\right)$ is a pointed cl-simple module, and constitutes a least element of the partially ordered set of such modules. In particular, that partially ordered set is nonempty.

Proof. Since $R / \mathrm{cl}_{R}(\{0\})$ is cl-semisimple, and is generated as a module by [1], the preceding lemma will give the first assertion if we can show that this module is nonzero, i.e., that $\mathrm{cl}_{R}(\{0\}) \neq R$.

Now the assumption that our coherent matroidal structure is proper says that for some $n, R^{n}$ has a proper cl-closed submodule $N$. Let $h: R \rightarrow R^{n}$ be a homomorphism taking 1 to an element not in $N$. Then $h^{-1}(N)$ is a proper clclosed submodule of $R$, so the least cl-closed submodule, $\mathrm{cl}_{R}(\{0\})$, is proper, as required.

The remaining assertions are straightforward.

Strictly speaking, we should have said that the chosen representative of the isomorphism class of $\left(R / \operatorname{cl}_{R}(\{0\}),[1]\right)$ is a least element of our partially ordered set; but we shall assume this sort of point understood; likewise in the statement of the next result.

Lemma 17. If $\left(M_{1}, p_{1}\right)$ and $\left(M_{2}, p_{2}\right)$ are pointed cl-simple modules, and we set

$$
\begin{aligned}
M & =M_{1} \oplus M_{2}, \\
M_{3} & =M / \operatorname{cl}_{M}\left(\left\{\left(p_{1},-p_{2}\right)\right\}\right), \\
p_{3} & =\text { image in } M_{3} \text { of }\left(p_{1}, 0\right), \text { equivalently, of }\left(0, p_{2}\right),
\end{aligned}
$$

then $\left(M_{3}, p_{3}\right)$ is again a pointed cl-simple module, and is a least upper bound of $\left(M_{1}, p_{1}\right)$ and $\left(M_{2}, p_{2}\right)$ in the partially ordered set of such modules.

In particular, the partially ordered set of pointed cl-simple modules is directed.

Proof. Note that the submodules $M_{1} \oplus\{0\}$ and $\{0\} \oplus M_{2}$ of $M$ are the kernels of homomorphisms to the cl-semisimple modules $M_{2}$ and $M_{1}$ respectively, hence are cl-closed. This shows, on the one hand, that their intersection $\{0\}$ is cl-closed, so that $M$ is cl-semisimple, and, on the other hand, that $M$ has nonzero proper closed 
submodules, so it is not cl-simple. Hence by Lemma 15] the closure of the singleton $\left\{\left(p_{1},-p_{2}\right)\right\}$ cannot be all of $M$. Thus $M_{3}$ is nonzero.

To show, next, that $\operatorname{cl}_{M_{3}}\left(\left\{p_{3}\right\}\right)$ is all of $M_{3}$, note that its inverse image in $M$ will contain both $\left(p_{1}, 0\right)$ and $\left(0, p_{2}\right)$. These are the images of $p_{1}$ and $p_{2}$ under the natural embeddings of $M_{1}$ and $M_{2}$ in $M$; hence the indicated inverse image module will contain the images of the closures of $\left\{p_{1}\right\}$ and $\left\{p_{2}\right\}$, which are all of $M_{1}$ and $M_{2}$. So that inverse image is all of $M$, so $\mathrm{cl}_{M_{3}}\left(\left\{p_{3}\right\}\right)=M_{3}$, proving cl-simplicity.

If we write $\left(M_{0}, p_{0}\right)$ for the initial pointed cl-simple module constructed in the previous lemma, we see that $M_{3}$ is the pushout in the category of cl-semisimple modules (as described in Lemma 12) of the unique distinguished-element-respecting maps $M_{0} \rightarrow M_{1}, M_{0} \rightarrow M_{2}$. The asserted characterization of $\left(M_{3}, p_{3}\right)$ as least upper bound to $\left(M_{1}, p_{1}\right)$ and $\left(M_{2}, p_{2}\right)$ follows.

Definition 18. The direct limit of the directed system of all pointed cl-simple $R$ modules will be denoted $D_{R}$. The element of this module which is the common image of the distinguished elements of these pointed modules will be denoted 1.

From the above definition and Lemma 13(ii), we have

Every pointed cl-simple module $(M, p)$ admits a unique homomorphism of pointed modules (i.e., module homomorphism respecting distinguished elements) to $\left(D_{R}, 1\right)$, and this homomorphism is oneto-one.

Note that $D_{R}$ is in general not finitely generated, hence is not, under our definition, a cl-simple module. However, the fact that it is a direct limit of such modules via one-to-one maps will allow us to apply to it what we know about such modules.

Let us now prove

Lemma 19. Every nonzero module endomorphism of $D_{R}$ is one-to-one, and for each nonzero element $d \in D_{R}$, there exists a unique endomorphism $m_{d}: D_{R} \rightarrow D_{R}$ carrying d to 1 .

Proof. The first assertion is clear from the definition of cl-simple module and our construction of $D_{R}$ as a direct limit of such modules, and implies that if there exists an endomorphism of $D_{R}$ taking a given element $d$ to 1 , it is unique.

For the proof of existence, we need some notation. Let $J$ be the directed partially ordered set of pointed cl-simple modules. Each $j \in J$, as an ordered pair, will be written $\left(M_{j}, p_{j}\right)$. For $j \leq k \in J, h_{j, k}$ will denote the unique distinguished-elementrespecting homomorphism $M_{j} \rightarrow M_{k}$, while for $j \in J, h_{j}$ will denote the unique distinguished-element-respecting homomorphism $M_{j} \rightarrow D_{R}$.

Now given nonzero $d \in D_{R}$, let us choose $j_{0} \in J$ and $x_{j_{0}} \in M_{j_{0}}$ such that $h_{j_{0}}\left(x_{j_{0}}\right)=d$. For each $j \geq j_{0}$, let $x_{j}=h_{j_{0}, j}\left(x_{j_{0}}\right) \in M_{j}$. Thus for each such $j$, $\left(M_{j}, x_{j}\right)$ is a pointed cl-simple module, and so by (22) there is a unique module homomorphism $m_{j}: M_{j} \rightarrow D_{R}$ sending $x_{j}$ to 1 . These homomorphisms to $D_{R}$ will form commuting triangles with the maps $h_{j, k}\left(j_{0} \leq j \leq k\right)$, for by construction, the required equalities are satisfied at the elements $x_{j}$, so by the unicity assertion of (22), they hold at all elements. Since these maps $m_{j}$ are defined for $j$ in a cofinal subset of $J$, and a direct limit of a cofinal subsystem of a directed system is the same as the direct limit of the whole system, they induce a map from the direct limit module $D_{R}$ to their common codomain $D_{R}$. This is the desired endomorphism $m_{d}$. 
Since $D_{R}$ is a right $R$-module, we shall write its endomorphisms on the left, and regard $D_{R}$ as a left module over $\operatorname{End}\left(D_{R}\right)$. From Lemma 19 we can easily deduce

Theorem 20. The endomorphism ring of $D_{R}$ is a division ring, over which $D_{R}$ is 1-dimensional as a left vector-space, with basis $\{1\}$.

Proof. If $a$ is a nonzero endomorphism of $D_{R}$, then by the first assertion of the preceding lemma it takes $1 \in D_{R}$ to a nonzero element $d$. By the second assertion of that lemma, there exists an endomorphism $b$ of $D_{R}$ taking $d$ to 1 ; hence $b a$ will be an endomorphism fixing 1 , hence by the unicity statement of the lemma, $b a$ is the identity endomorphism. So every nonzero element of $\operatorname{End}\left(D_{R}\right)$ is left invertible, so $\operatorname{End}\left(D_{R}\right)$ is a division ring.

From the preceding lemma and the above invertibility observations, we see that the group of nonzero elements of $\operatorname{End}\left(D_{R}\right)$ will act transitively on the set of nonzero elements $d \in D_{R}$, hence $D_{R}$ must be one-dimensional as a left vector space over this division ring. Since $1 \in D_{R}$ is nonzero, $\{1\}$ is a basis.

Thus, the map $a \mapsto a(1)$ is a bijection between elements of $\operatorname{End}\left(D_{R}\right)$ and of $D_{R}$. This allows us to transfer the structure of division ring of the former set onto the latter. The reader should verify that the resulting operations are as described in the next definition.

Definition 21. We shall denote by $D$ the division ring having the underlying additive group of $D_{R}$, and with multiplication such that for $d, e \in D_{R}$, the product de is the element obtained by applying to e the unique $R$-module endomorphism of $D_{R}$ which carries 1 to d (the inverse of the endomorphism constructed in Lemma 19 if $d$ is nonzero; the zero endomorphism otherwise).

The set-map $R \rightarrow D$ taking $r \in R$ to $1 r$ (product defined using the right $R$-module structure of $D_{R}$ ) will be denoted $f$.

Note that the multiplicative identity element of the above division ring will be the element we have named $1 \in D_{R}$.

Theorem 22. (i) The map $f: R \rightarrow D$ is a ring homomorphism, with kernel $\operatorname{cl}_{R}(\{0\})$.

(ii) The original right $R$-module structure of $D_{R}$ is the same as the right $R$ module structure induced on the division ring $D$ by this homomorphism $f$.

(iii) $D$ is generated as a division ring by the subring $f(R)$.

Proof. That $f$ respects addition is clear; from Lemma 16 we see that it has kernel $\operatorname{cl}_{R}(\{0\})$, and by construction it takes 1 to 1 .

In proving that it respects multiplication and satisfies (ii), let us temporarily write - for the internal multiplication of $D$ specified in Definition 21, while continuing to use juxtaposition to denote the internal multiplication of $R$ and the right $R$-module structure of $D_{R}$. By Definition 21, left multiplication by any fixed element $d \in D$ acts by an $R$-module endomorphism that takes 1 to $d$. Hence for any such $d$, and any $r \in R$,

$$
d \cdot f(r)=d \cdot(1 r)=d r .
$$

Now if in (23) we take for $d$ an element $f(s)=1 s(s \in R)$, we get $f(s) \cdot f(r)=$ $(1 s) r=1(s r)=f(s r)$, so $f$ respects multiplication, completing the proof of (i). Since $D_{R}$ and $D$ have the same additive structure, (23) is now equivalent to (ii). 
To prove (iii), suppose that $f(R)$ were contained in a smaller division $\operatorname{ring} E \subset D$. Then as a right $E$-vector-space, $D$ would have dimension $>1$, hence it would have a nontrivial direct sum decomposition, which by (ii) would be a direct sum decomposition of $D_{R}$ as an $R$-module. But the existence of such a decomposition would imply that $\operatorname{End}\left(D_{R}\right)$ had zero divisors, contradicting Theorem 20 .

This completes the promised construction!

Let us note a few facts about the $R$-module structure of $D_{R}$. In view of its construction as a direct limit, every finitely generated $R$-submodule of $D_{R}$ lies in a cl-simple (hence cl-semisimple) submodule, hence is cl-semisimple. Suppose $M_{1}$ and $M_{2}$ are cl-simple submodules of $D_{R}$, both containing 1 . Then the sum $M_{1}+M_{2}$ will be a nonzero cl-semisimple module generated by images of $M_{1}$ and $M_{2}$, in which the copies of the element 1 are identified. It easily follows that $\left(M_{1}+M_{2}, 1\right)$ must be isomorphic to the object constructed as in Lemma 17 from the pointed cl-simple modules $\left(M_{1}, 1\right)$ and $\left(M_{2}, 1\right)$; that is, $\left(M_{1}+M_{2}, 1\right)$ is, up to isomorphism, the least upper bound of those elements in the directed partially ordered set of pointed clsimple $R$-modules. From this we see that the submodule of $D_{R}$ generated by any family of cl-simple submodules each of which contains 1 is the direct limit of some directed subsystem of the system we used to construct $D_{R}$. We record for later use a property of such submodules.

Lemma 23. Suppose $C_{R} \subseteq D_{R}$ is the direct limit of a nonempty directed subsystem of the directed system of cl-simple modules used above in constructing $D_{R}$. Then the restriction map $\operatorname{Hom}\left(D_{R}, D_{R}\right) \rightarrow \operatorname{Hom}\left(C_{R}, D_{R}\right)$ is an isomorphism. In particular, every endomorphism of $C_{R}$ extends uniquely to an endomorphism of $D_{R}$, so $\operatorname{End}\left(C_{R}\right)$ can be identified with a subring of the division ring $\operatorname{End}\left(D_{R}\right) \cong D$.

Proof. That the restriction map $\operatorname{Hom}\left(D_{R}, D_{R}\right) \rightarrow \operatorname{Hom}\left(C_{R}, D_{R}\right)$ is one-to-one follows merely from the facts that $\operatorname{End}\left(D_{R}\right)$ is a division ring, and $C_{R}$ a nonzero $R$-submodule of $D_{R}$. To show that this map is surjective, let $h \in \operatorname{Hom}\left(C_{R}, D_{R}\right)$. If we write $h^{\prime} \in \operatorname{Hom}\left(C_{R}, D_{R}\right)$ for the restriction to $C_{R}$ of the unique endomorphism of $D_{R}$ taking 1 to $h(1)$, then $\left(h-h^{\prime}\right)(1)=0$, whence by the final assertion of (22) and the hypothesis on $C_{R}, h=h^{\prime}$, as required.

The first half of the last sentence of the lemma is seen by regarding $\operatorname{End}\left(C_{R}\right)$ as a subset of $\operatorname{Hom}\left(C_{R}, D_{R}\right)$; the remaining assertion clearly follows.

\section{TYING UP LOOSE ENDS}

We saw in $\$ 2$ that a homomorphism $f$ from a ring $R$ to a division ring $D$ induces a coherent matroidal structure on free $R$-modules; we have just seen how to construct from such a matroidal structure a homomorphism into a division ring. It remains to prove that when we restrict the former construction to homomorphisms such that $D$ is generated as a division ring by $f(R)$, the two constructions become inverse to one another.

As in the last two sections, whenever we introduce a family $\left(\mathrm{cl}_{R^{n}}\right)_{n \in \mathbf{N}}$ satisfying the conditions of (14), we shall understand this family to be extended via Lemma 9 to operators $\mathrm{cl}_{M}$ on general finitely generated $R$-modules $M$.

A key step in proving our constructions to be inverses will be a comparison of two characterizations of semisimple $R$-modules. The first of these is 
Lemma 24. Let $R$ be a ring, $\left(\mathrm{cl}_{R^{n}}\right)_{n \in \mathbf{N}}$ a coherent matroidal structure on free $R$ modules, and $D$ the division ring of Definition [21] given with the $R$-module structure of Theorem 22(ii).

Then for any finitely generated $R$-module $M$, the following conditions are equivalent:

(i) $M$ is cl-semisimple.

(ii) $M$ is embeddable in a direct product of (possibly infinitely many) cl-simple $R$-modules.

(iii) $M$ is embeddable as an $R$-module in a (possibly infinite-dimensional) vector space over $D$.

Proof. The implications $(\mathrm{i}) \Leftarrow($ ii $) \Leftrightarrow($ iii) are straightforward, the first by $(20)$, the remaining double implication using the fact that $D_{R}$ is a direct limit of "all" clsimple $R$-modules, and the consequence that every finitely generated submodule of $D_{R}$ is contained in a cl-simple submodule. To prove (i) $\Rightarrow($ ii), it will suffice to show that if (i) holds, and $z$ is a nonzero element of $M$, then there exists a homomorphism from $M$ to a cl-simple module not having $z$ in its kernel.

To do this, let $X$ be a finite subset of $M$ of least cardinality such that $\operatorname{cl}_{M}(X)=$ $M$, and $X_{0}$ a minimal subset of $X$ such that $z \in \operatorname{cl}_{M}\left(X_{0}\right)$. Since $M$ is semisimple and $z \neq 0, X_{0}$ is nonempty. Now from the exchange axiom (5) it follows that in $X_{0}$, we can replace any element $y$ by $z$ without changing the closure; hence in $X$ we can replace any element of $X_{0}$ by $z$ without changing the closure; thus we can assume $z \in X$. By the minimality of $\operatorname{card}(X), z \notin \mathrm{cl}_{M}(X-\{z\})$; calling the latter submodule $N$, we see that $M / N$ is cl-semisimple, and is the closure of the image of $z$, hence is cl-simple. The canonical map $M \rightarrow M / N$ is the required homomorphism.

(Incidentally, one can deduce from the fact that $M$ is a finitely generated module, hence finitely generated as a matroid, that in (ii) and (iii) above it suffices to refer to finite products and finite-dimensional vector spaces. But we will not need this fact.)

Our next result, which we will play off against the preceding one, concerns the system of closure operators induced by a ring homomorphism.

Lemma 25. Suppose $f: R \rightarrow S$ is a ring homomorphism, and $\left(\mathrm{cl}_{R^{n}}\right)_{n \in \mathbf{N}}$ is the family of closure operators on free $R$-modules induced by $f$ as in Corollary 8

Then for any finitely generated $R$-module $M$, the cl-closed submodules of $M$ are precisely the kernels of $R$-module homomorphisms of $M$ into $S$-modules (regarded as $R$-modules via the ring homomorphism $f$ ). Thus, $M$ is cl-semisimple if and only if it is embeddable in an S-module; equivalently, if and only if the extension-of-scalars map $M \rightarrow M \otimes_{R} S$ is one-to-one.

Proof. Straightforward from the definition of $\left(\mathrm{cl}_{R^{n}}\right)_{n \in \mathbf{N}}$.

From the above two lemmas, we can now prove

Proposition 26. Let $\left(\mathrm{cl}_{R^{n}}\right)_{n \in \mathbf{N}}$ be a coherent matroidal structure on free $R$ modules, and $f: R \rightarrow D$ the homomorphism to a division ring constructed therefrom as in the preceding section. Then the coherent matroidal structure on free $R$-modules induced as in Corollary 8 by this map $f$ is again $\left(\mathrm{cl}_{R^{n}}\right)_{n \in \mathbf{N}}$.

Proof. Comparing the characterization in Lemma $24(\mathrm{i}) \Leftrightarrow($ iii) of $R$-modules semisimple with respect to our given matroidal structure, and the characterization in 
Lemma 25 of $R$-modules semisimple under the matroidal structure induced by $f$, we see that these both consist of the modules embeddable over $R$ in right $D$-vectorspaces.

But I claim that a coherent matroidal structure on free $R$-modules, or more generally, a system $\left(\mathrm{cl}_{R^{n}}\right)_{n \in \mathbf{N}}$ of closure operators as in (14) , is determined by its class of semisimple modules. Indeed, the cl-semisimple modules determine the clclosed subsets of $R^{n}$, since $N \subseteq R^{n}$ is cl-closed if and only if $R^{n} / N$ is cl-semisimple; and any closure operator on a set is determined by the class of closed subsets, since the operator sends each subset to the least closed subset containing it.

Hence the two matroidal structures in question are equal.

It remains to show that the composite of our constructions in the opposite order, division ring to coherent matroidal structure to division ring, is also isomorphic to the identity. For this we will use the following criterion for division rings to be isomorphic over $R$ :

Lemma 27. Let $R$ be a ring, and $f_{1}: R \rightarrow D_{1}, f_{2}: R \rightarrow D_{2}$ homomorphisms into division rings. Then the division subring of $D_{1}$ generated by $f_{1}(R)$ and the division subring of $D_{2}$ generated by $f_{2}(R)$ are isomorphic as rings with homomorphisms of $R$ into them if and only if the abelian group $D_{1} \otimes_{R} D_{2}$ is nonzero; equivalently, if and only if $1 \otimes 1 \neq 0$ in that group.

Proof. If the division subrings of $D_{1}$ and $D_{2}$ generated by the images of $R$ are isomorphic over $R$, then identifying them, and calling their common value $D$, we can map the abelian group $D_{1} \otimes_{R} D_{2}$ onto $D_{1} \otimes_{D} D_{2}$ by $x \otimes_{R} y \mapsto x \otimes_{D} y$; and the latter abelian group, being a tensor product of nonzero vector spaces (right and left respectively) over a common division ring, is nonzero. So we have the implication " $\Rightarrow$ ".

To prove the converse, consider the set of pairs

$$
i=\left\{\left(d_{1}, d_{2}\right) \in D_{1} \times D_{2} \mid d_{1} \otimes 1=1 \otimes d_{2} \text { in } D_{1} \otimes_{R} D_{2}\right\} .
$$

Note that if $i$ contained distinct pairs with the same second component, $\left(d_{1}, d_{2}\right)$ and $\left(d_{1}^{\prime}, d_{2}\right)$, then we would have $\left(d_{1}-d_{1}^{\prime}\right) \otimes 1=0$, hence, recalling that that the abelian group $D_{1} \otimes D_{2}$ has a natural structure of $\left(D_{1}, D_{2}\right)$-bimodule, and multiplying on the left by $\left(d_{1}-d_{1}^{\prime}\right)^{-1}$, we would have $1 \otimes 1=0$. So assuming the contrary, each second component corresponds to at most one first component, and by the symmetric argument, each first component corresponds to at most one second component. Thus, $i$ is the graph of a bijection between a subset of $D_{1}$ and a subset of $D_{2}$.

Now let $\left(d_{1}, d_{2}\right)$ and $\left(d_{1}^{\prime}, d_{2}^{\prime}\right)$ be any two elements of $i$. We see from the linearity of $\otimes$ in each argument that $i$ will also contain $\left(d_{1}+d_{1}^{\prime}, d_{2}+d_{2}^{\prime}\right)$, so our subsets of $D_{1}$ and $D_{2}$ are each closed under addition and our bijection respects this operation. To get the corresponding conclusion for multiplication, we again use the $\left(D_{1}, D_{2}\right)$ bimodule structure of $D_{1} \otimes D_{2}$ :

$$
\begin{aligned}
\left(d_{1} d_{1}^{\prime}\right) \otimes 1 & =d_{1}\left(d_{1}^{\prime} \otimes 1\right)=d_{1}\left(1 \otimes d_{2}^{\prime}\right)=d_{1} \otimes d_{2}^{\prime} \\
& =\left(d_{1} \otimes 1\right) d_{2}^{\prime}=\left(1 \otimes d_{2}\right) d_{2}^{\prime}=1 \otimes\left(d_{2} d_{2}^{\prime}\right),
\end{aligned}
$$

showing that $\left(d_{1} d_{1}^{\prime}, d_{2} d_{2}^{\prime}\right) \in i$. Similarly, if $(0,0) \neq\left(d_{1}, d_{2}\right) \in i$, then multiplying the equation $d_{1} \otimes 1=1 \otimes d_{2}$ by $d_{1}^{-1}$ on the left and $d_{2}^{-1}$ on the right, we get $\left(d_{1}^{-1}, d_{2}^{-1}\right) \in i$.

We also note that by definition of the tensor product, $i$ contains all pairs $\left(f_{1}(r)\right.$, $\left.f_{2}(r)\right)(r \in R)$. 
The above results together say that $i$ is the graph of an isomorphism between a division subring of $D_{1}$ and a division subring of $D_{2}$, under which elements $f_{1}(r)$ correspond to elements $f_{2}(r)$. Thus, the division subrings generated by the respective images of $R$ are isomorphic over $R$.

We can now complete the proof that our constructions are inverse to one another by proving

Proposition 28. Let $f_{1}: R \rightarrow D_{1}$ be a homomorphism from a ring to a division ring, let $\left(\mathrm{cl}_{R^{n}}\right)_{n \in \mathbf{N}}$ be the coherent matroidal structure on free $R$-modules determined by $f_{1}$ as in Corollary [8, and let $f_{2}: R \rightarrow D_{2}$ be the homomorphism into a division ring constructed from that coherent matroidal structure as in Theorem 22. Then the division subring of $D_{1}$ generated by $f_{1}(R)$ is isomorphic to $D_{2}$ as division rings with homomorphisms of $R$ into them.

Proof. $D_{2}$ was constructed as a direct limit of one-to-one homomorphisms of clsimple (hence cl-semisimple) $R$-modules, and by Lemma 25 for each such $R$-module $M$, the canonical map $M \rightarrow M \otimes_{R} D_{1}$ is an embedding. Hence the canonical map $D_{2} \rightarrow D_{2} \otimes_{R} D_{1}$ is also an embedding. The preceding lemma, and the fact that $D_{2}$ is generated by $f_{2}(R)$ now yield the asserted conclusion.

Though we shall not need the fact, it is interesting to note

Lemma 29. In the context of Lemma 27, the condition $D_{1} \otimes_{R} D_{2} \neq\{0\}$ is also equivalent to the condition $\operatorname{Hom}_{\mathbf{M o d}_{R}}\left(D_{1}, D_{2}\right) \neq\{0\}$.

Proof. $\operatorname{Hom}_{\operatorname{Mod}_{R}}\left(D_{1}, D_{2}\right) \cong \operatorname{Hom}_{\operatorname{Mod}_{D_{2}}}\left(D_{1} \otimes_{R} D_{2}, D_{2}\right)$, and by standard properties of vector spaces, this abelian group is nonzero if and only if the $D_{2}$-vector space $D_{1} \otimes_{R} D_{2}$ is nonzero.

(It might be of interest to consider a ring $R$ with homomorphisms $R \rightarrow S_{1}$, $R \rightarrow S_{2}$ where $S_{1}$ and/or $S_{2}$ belong to classes of rings wider than or different from the class of division rings, and see what relations between these two objects can be expressed in terms of properties of $S_{1} \otimes_{R} S_{2}$ or $\operatorname{Hom}_{M_{\text {od }}}\left(S_{1}, S_{2}\right)$.)

\section{EASY EXAMPLES: DIVISION RINGS OF FRACTIONS OF ORE RINGS}

Recall that a right (respectively left) Ore ring is a nonzero ring without zerodivisors in which any two nonzero elements have a nonzero common right (respectively left) multiple. These are the rings for which there exist simple classical constructions of embeddings in division rings. And in fact, we can characterize rings of these two sorts by statements that they admit coherent matroidal structures with particular natural descriptions.

Lemma 30. Let $R$ be a nonzero ring without zero-divisors, and for all $n \in \mathbf{N}$ and $X \subseteq R^{n}$ let

$$
\operatorname{cl}_{R^{n}}(X)=\left\{y \in R^{n} \mid(\exists r \in R-\{0\}) y r \in X R\right\} .
$$

Then the following conditions are equivalent:

(i) For all $n \in \mathbf{N}, \mathrm{cl}_{R^{n}}$ is a closure operator on $R^{n}$.

(ii) For all $n \in \mathbf{N}$ and $X \subseteq R^{n}, \operatorname{cl}_{R^{n}}(X)$ is closed under addition.

(iii) For all $n \in \mathbf{N}$ and $X \subseteq R^{n}, \operatorname{cl}_{R^{n}}(X)$ is closed under right multiplication by elements of $R$.

(iv) $\left(\mathrm{cl}_{R^{n}}\right)_{n \in \mathbf{N}}$ is a coherent matroidal structure on free $R$-modules. 
(v) $R$ is a right Ore ring.

When these equivalent conditions hold, the division ring constructed from $\left(\mathrm{cl}_{R^{n}}\right)_{n \in \mathbf{N}}$ as $\$$ 政 the classical division ring of right fractions of $R$.

Proof. Let us first show that assuming (v), the coherent matroidal structure induced by the homomorphism of $R$ into its division ring $D$ of right fractions is described by (24). Given $X \subseteq R^{n}$ the closure of $X$ with respect to the matroidal structure induced by $D$ consists of all elements $y \in R^{n}$ such that in $D^{n}$ we can write $y=$ $\sum x_{i} d_{i}$ for some $x_{1}, \ldots, x_{n} \in X, d_{1}, \ldots, d_{n} \in D$. Since $R$ is right Ore, we can bring $d_{1}, \ldots, d_{n}$ to a common right denominator, and so write this relation $y=\sum x_{i} s_{i} r^{-1}$ with $s_{i} \in R, r \in R-\{0\}$. The existence of such a relation is equivalent to saying that $y r \in X R$ for some $r \in R-\{0\}$, as required.

This yields the implication (v) $\Rightarrow($ iv), and (pending the equivalence of the other conditions with $(\mathrm{v}))$ the final assertion of the lemma. Clearly (iv) $\Rightarrow(\mathrm{i})$, and since $\mathrm{cl}_{R^{n}}(X) \supseteq X R,(\mathrm{i}) \Rightarrow(\mathrm{ii}) \wedge(\mathrm{iii})$. The proof will be complete if we can show that each of (ii) and (iii) implies (v).

Assuming (ii) or (iii), let $a, b \in R-\{0\}$; we want to prove that these elements have a nonzero common right multiple in $R$. First take $n=1$ and $X=\{a\}$. Then $1 \in \operatorname{cl}_{R}(\{a\})$, so (iii) implies that $b \in \operatorname{cl}_{R}(\{a\})$. Applying the definition (24) of $\mathrm{cl}_{R}$, we get the desired common multiple assertion. Likewise, if $n=2$ and $X=$ $\{(a, 0),(0, b)\}$, then $(1,0),(0,1) \in \mathrm{cl}_{R^{2}}(X)$, so (ii) implies that $(1,1) \in \operatorname{cl}_{R^{2}}(X)$, which by (24) again says that $a$ and $b$ have a nonzero common right multiple.

In the above lemma, we found that if the operators $\mathrm{cl}_{R^{n}}$ we defined had any of the most elementary desired properties, then they had them all, and $R$ was right Ore. In the next lemma, on the other hand, where we define the operators appropriate to left Ore rings, we shall find that these automatically have most of the desired properties, and that the Ore condition is equivalent to the final key property.

Lemma 31. Let $R$ be any nonzero ring, and for $n \in \mathbf{N}$ and $X \subseteq R^{n}$ let (25) $\operatorname{cl}_{R^{n}}(X)=\bigcap\left\{\operatorname{Ker} h \mid h: R^{n} \rightarrow R\right.$ is an $R$-linear map with $X$ in its kernel $\}$.

Then $\left(\mathrm{cl}_{R^{n}}\right)_{n \in \mathbf{N}}$ satisfies (1)-(3), (6) and (7); and the following conditions are equivalent:

(i) $\left(\mathrm{cl}_{R^{n}}\right)_{n \in \mathbf{N}}$ also satisfies (5) (and hence, by Lemma 2, (4)); i.e., constitutes a coherent matroidal structure on free $R$-modules.

(ii) $R$ is a left Ore ring.

When these equivalent conditions hold, the division ring constructed from $\left(\mathrm{cl}_{R^{n}}\right)_{n \in \mathbf{N}}$ as in $\$ 4$ is the classical division ring of left fractions of $R$.

Proof. That (25) satisfies (1)-(3), (6) and (7) is straightforward. Also note that if a ring $R$ is embedded in a division ring $D$, and we regard $R^{n}$ as a subset of $D^{n}$, then by elementary linear algebra, the closure of $X \subseteq R^{n}$ with respect to the coherent matroidal structure on $R$-modules determined by $D$ is equal to the intersection of $R^{n}$ with the kernels of all $D$-linear maps $h: D^{n} \rightarrow D$ having $X$ in their kernels. If we now assume (ii), and let $D$ be the division ring of left fractions of $R$, then for each such $D$-linear map $h$, we can bring a finite set of generators of the $R$ submodule $h\left(R^{n}\right) \subseteq D$ to a common left denominator, getting $h\left(R^{n}\right) \subseteq r^{-1} R$ for some $r \in R-\{0\}$. Now composing $h$ with the right vector space endomorphism of $D$ given by left multiplication by $r$, we get a $D$-linear map with the same kernel which carries $R^{n}$ into $R$, and hence restricts to an $R$-linear map $h^{\prime}: R^{n} \rightarrow R$. 
This shows that the closure operator induced by $D$ is indeed (25), yielding the implication (ii) $\Rightarrow$ (i) and (pending the reverse implication) the final assertion.

To show (i) $\Rightarrow$ (ii), let us first note that the operator defined by (25) for $n=1$ satisfies $\operatorname{cl}_{R}(\varnothing)=\{0\}$ and $\operatorname{cl}_{R}(\{1\})=R$. Hence (5) implies that for every nonzero $a \in R$ we have $1 \in \operatorname{cl}_{R}(\{a\})$. Thus, every $R$-linear functional on $R$, i.e., every left multiplication operation, if it annihilates $a$, also annihilates 1, i.e., is zero; which shows that $R$ has no zero divisors. Second, taking $n=2$ and $a, b \in R-\{0\}$, consideration of the first-coordinate functional shows that the operator (25) satisfies $\left.(a, b) \notin \operatorname{cl}_{R^{2}}(\{0,1)\}\right)$. Hence (5) gives $(0,1) \notin \operatorname{cl}_{R^{n}}(\{(a, b)\})$, so there is some $R$ linear functional $h$ annihilating $(a, b)$ but not $(0,1)$. The relation $0=h((a, b))=$ $h((1,0)) a+h((0,1)) b$ now shows that $R$ is left Ore.

In proving the above lemmas, we called on the known result that right and left Ore rings have division rings of right and left fractions. We could instead have proved directly that for such rings, the definitions (24) and (25) yielded coherent matroidal structures on free $R$-modules, and hence embeddings in division rings; but we wanted to establish the connection with the elegant standard constructions. The next section, on the other hand, will give a class of examples for which embeddability in division rings was not known until Cohn proved it by describing the corresponding prime matrix ideals; we shall likewise prove it by describing the corresponding coherent matroidal structures.

\section{Application: the Universal Division Ring OF A FiR}

Recall that a free right ideal ring or right fir is a ring $R$ such that every right ideal of $R$ is free as a right $R$-module, and such that $R^{m} \cong R^{n}$ as right $R$-modules only when $m=n$ [5], [7]. In this section we will assume $R$ a right fir; "module" will continue to mean "right module".

If $F$ is a finitely generated free $R$-module, we shall write $\operatorname{rank}(F)$ for the unique $n$ such that $F \cong R^{n}$. From the freeness of right ideals, one easily deduces that submodules of free $R$-modules are also free. Below, we will regularly speak of the rank of a finitely generated submodule $M$ of a free $R$-module $F$ without an explicit reminder that this makes sense for the above reason.

If $M$ and $M^{\prime}$ are finitely generated submodules of a free $R$-module $F$, then the standard short exact sequence $\{0\} \rightarrow M \cap M^{\prime} \rightarrow M \oplus M^{\prime} \rightarrow M+M^{\prime} \rightarrow\{0\}$ splits, by the freeness of $M+M^{\prime}$. Hence $M \cap M^{\prime}$ is finite generated, and

$$
\operatorname{rank}\left(M \cap M^{\prime}\right)+\operatorname{rank}\left(M+M^{\prime}\right)=\operatorname{rank}(M)+\operatorname{rank}\left(M^{\prime}\right) .
$$

Now let $X$ be a subset of $R^{n}$ for some $n \in \mathbf{N}$, and let $m \leq n$ be the least among the ranks of all finitely generated submodules of $R^{n}$ containing $X$. (This may be less than $\operatorname{rank}(X R)$. For instance, if $x$ is a nonzero element of $R^{n}$, and $s, t$ are right $R$-linearly independent elements of $R$, then taking $X=\{x s, x t\}, X R$ has rank 2, but is contained in $x R$, so $m=1$.) It is easy to see from (26) that the sum of any two submodules of $F$ that contain $X$ and each have this least rank $m$ will also have rank $m$. Let $\mathrm{cl}_{R^{n}}(X)$ denote the sum of all submodules of $R^{n}$ that contain $X$ and have rank $m$.

I claim that $\mathrm{cl}_{R^{n}}(X)$ is finitely generated. To see this, choose any rank- $m$ submodule $M_{0} \subseteq R^{n}$ containing $X$. Taking a free generating set (possibly infinite) for $\mathrm{cl}_{R^{n}}(X)$, we see that the finitely generated submodule $M_{0}$ can involve only finitely many members of that generating set, hence will lie in a finite-rank direct summand 
$N \subseteq \operatorname{cl}_{R^{n}}(X)$. If $N$ were a proper summand, then since $\operatorname{cl}_{R^{n}}(X)$ is a directed union of submodules of rank $m$ containing $X$, we could find a rank- $m$ submodule $M_{1}$ properly containing $N$. Then $N$ would also be a proper direct summand of $M_{1}$, hence would have $\operatorname{rank}<\operatorname{rank}\left(M_{1}\right)=m$, contradicting our choice of $m$. So we must have $N=\operatorname{cl}_{R^{n}}(X)$, proving the latter finitely generated, as claimed. Hence, being a directed union of free modules of rank $m$, it must be one itself. So $\operatorname{cl}_{R^{n}}(X)$ is the largest submodule of $R^{n}$ containing $X$ and having this rank.

I claim now that the family of operators $\mathrm{cl}_{R^{n}}$ so defined satisfies (1)-(7).

(1), (3), and (6) are clear. To see (2), let $X \subseteq Y$, and let us apply (26) with $M=\operatorname{cl}_{R^{n}}(X)$ and $M^{\prime}=\operatorname{cl}_{R^{n}}(Y)$. The minimality assumption on the rank of $M=\operatorname{cl}_{R^{n}}(X)$ shows that $M \cap M^{\prime}$ will have at least that rank, hence $M+M^{\prime}$ will have at most the rank $M^{\prime}=\operatorname{cl}_{R^{n}}(Y)$. The minimality condition on that rank, and the fact that $M^{\prime}$ is the largest submodule containing $Y$ and having that rank, now show that $M+M^{\prime}=M^{\prime}$, i.e., that $M \subseteq M^{\prime}$, as required.

To see (4), recall that for $Y \subseteq R^{n}, \operatorname{rank}\left(\operatorname{cl}_{R^{n}}(Y)\right) \leq n$. Hence given $X \subseteq R^{n}$, we can choose among all finite subsets $X_{0} \subseteq X$ one that maximizes $\operatorname{rank}\left(\operatorname{cl}_{R^{n}}\left(X_{0}\right)\right)$. We see easily that for every finite subset $X_{1} \subseteq X$ containing $X_{0}, \operatorname{cl}_{R^{n}}\left(X_{1}\right)=\operatorname{cl}_{R^{n}}\left(X_{0}\right)$. Hence $X \subseteq \operatorname{cl}_{R^{n}}\left(X_{0}\right)$, so by (21) and (3) we have $\operatorname{cl}_{R^{n}}(X)=\operatorname{cl}_{R^{n}}\left(X_{0}\right)$, giving (4).

To prove (5), let $X, y$ and $z$ be as in the hypothesis of that condition. Let $m=\operatorname{rank}\left(\operatorname{cl}_{R^{n}}(X)\right)$. Since $\operatorname{cl}_{R^{n}}(X \cup\{y\})$ is properly larger than $\operatorname{cl}_{R^{n}}(X)$, it must (by definition of $\mathrm{cl}_{R^{n}}(X)$ ) have larger rank. On the other hand, since $X \cup\{y\}$ is contained in the submodule $\operatorname{cl}_{R^{n}}(X)+y R$ of rank at most $m+1$, the rank of $\mathrm{cl}_{R^{n}}(X \cup\{y\})$ can't be larger than $m+1$; so that is precisely its value. By the same argument $\operatorname{cl}_{R^{n}}(X \cup\{z\})$ has rank $m+1$. But by assumption, $\operatorname{cl}_{R^{n}}(X \cup\{y\})$ contains $z$, hence contains $\operatorname{cl}_{R^{n}}(X \cup\{z\})$, so by the maximality condition in the definition of the latter, these must be equal, giving (15).

To prove (7), let $h$ and $X$ be as in the hypothesis of that statement. Since any submodule of $R^{n}$ is free, if we restrict the domain and codomain of $h$ to get a surjective homomorphism

$$
h^{\prime}: \operatorname{cl}_{R^{m}}(X) \rightarrow h\left(\operatorname{cl}_{R^{m}}(X)\right),
$$

this map splits. Now if the desired inclusion $h\left(\operatorname{cl}_{R^{m}}(X)\right) \subseteq \mathrm{cl}_{R^{n}}(h(X))$ fails, then $\mathrm{cl}_{R^{n}}(h(X))+h\left(\mathrm{cl}_{R^{m}}(X)\right)$ must have larger rank than $\mathrm{cl}_{R^{n}}(h(X))$ (again by the definition of that closure), hence by (26), $\operatorname{cl}_{R^{n}}(h(X)) \cap h\left(\operatorname{cl}_{R^{m}}(X)\right)$ must have smaller rank than $h\left(\mathrm{cl}_{R^{m}}(X)\right)$. Taking the inverse image of this submodule under the split surjection $h^{\prime}$, we get a submodule of $\operatorname{cl}_{R^{m}}(X)$ containing $X$ and having smaller rank than $\mathrm{cl}_{R^{m}}(X)$, a contradiction.

This shows that the above system of closure operators constitutes a coherent matroidal structure on free $R$-modules; hence the construction of $\$ 4$ leads to a homomorphism $f$ of $R$ into a division ring $D$. Clearly, $\operatorname{cl}_{R}(\{0\})=\{0\}$, so $f$ is an embedding.

The resulting division rings $D$ have been studied by Cohn [7] and others. Cohn constructs the corresponding prime matrix ideal (cf. \$1 above) as the set of all square matrices over $R$ which can be factored into a matrix with fewer columns times a matrix with fewer rows. (Cohn also briefly describes in [5, pp. 241 (bottom) to 243] the direct-limit approach of this paper, communicated to him by the present author, for the case of firs, without the general matroid formulation.)

Free associative algebras over fields are examples of firs, and as we mentioned in the Introduction, such free algebras in general admit many embeddings in division 
rings. However, Cohn [7, §4.5] shows (and we will see toward the end of the next section) that for any fir $R$ the map $R \rightarrow D$ just obtained is characterized by a universal property.

The above construction of a coherent matroidal structure on free modules over a fir admits straightforward generalizations in two directions, for which the interested reader can easily work out the details (or cf. [7, [13]). On the one hand, we can weaken the assumption that all right ideals of $R$ are free to say that all finitely generated right ideals are free, i.e., replace our right fir by a semifir. In this case, we cannot say, after defining $\operatorname{cl}_{R^{n}}(X)$ as above, that this submodule is finitely generated, and hence free of rank $m$; but clearly it is still locally free of rank $m$ (meaning that every finite subset is contained in a free submodule of rank $m$, and some finite subset is not contained in any free submodule of smaller rank); and we can use this in our remaining arguments, replacing calculations involving this module with calculations involving "sufficiently large" submodules; or, essentially equivalently, generalizing (26) to a statement about "local ranks" of submodules.

On the other hand, we can replace the assumption that $R$ is a right (semi)fir by the condition that it is right (semi)hereditary, i.e, that all its right ideals (respectively, finitely generated right ideals) are projective as right modules, and also assume that it admits an additive function from isomorphism classes of nonzero finitely generated projective modules to positive integers, which takes the free module on one generator to 1. Regarding such a function as defining the "rank" of a finitely generated projective module, we can duplicate the above construction. Such a projective rank function is not in general unique; when a semihereditary ring $R$ admits different rank functions, these determine different division rings $D$.

There are also generalizations of the above construction which cannot be so briefly summarized; cf. [13, Chapter 5].

For some results on a different class of modules over firs that use arguments of a similar flavor to those of this section, see [5, $\S \S 3.3,5.3]$.

\section{Specializations}

Given closure operators $\mathrm{cl}$ and $\mathrm{cl}^{\prime}$ on a set $F$, it is natural to write $\mathrm{cl} \leq \mathrm{cl}^{\prime}$ if for all $X \subseteq F, \operatorname{cl}(X) \subseteq \operatorname{cl}^{\prime}(X)$. Using this definition, we find that if we take a ring homomorphism $S \rightarrow T$ and let $\mathrm{cl}_{S^{n}}=\mathrm{cl}_{S^{n}}^{\bmod }$ (as defined in Lemma 5), while we let $\mathrm{cl}_{S^{n}}^{\prime}$ be the closure operator on $S^{n}$ induced via $f$ from $\mathrm{cl}_{T^{n}}^{\text {mod }}$ as in Corollary $\mathbb{8}$, then $\mathrm{cl}_{S^{n}} \leq \mathrm{cl}_{S^{n}}^{\prime}$. From this we can see, more generally, that for a ring $R$, if $\mathrm{cl}_{R^{n}}, \mathrm{cl}_{R^{n}}^{\prime}$ are the closure operators on $R^{n}$ induced via ring homomorphisms $R \rightarrow S, R \rightarrow T$ from the respective operators $\mathrm{cl}_{S^{n}}^{\bmod }, \mathrm{cl}_{T^{n}}^{\bmod }$, and if there exists a homomorphism $S \rightarrow T$ making a commuting triangle with these two homomorphisms from $R$, then $\mathrm{cl}_{R^{n}} \leq \mathrm{cl}_{R^{n}}^{\prime}$.

However, given homomorphisms from a ring $R$ to two division rings $D_{1}$ and $D_{2}$ generated in nonisomorphic ways by the images of $R$, there can be no homomorphism between $D_{1}$ and $D_{2}$ making such a commuting triangle; so we should not expect such inequalities between the induced coherent matroidal structures. For example, for $R=\mathbf{Z}$, if we define $\mathrm{cl}_{\mathbf{Z}^{n}}$ and $\mathrm{cl}_{\mathbf{Z}^{n}}^{\prime}$ to be the coherent matroidal structures induced by the homomorphisms $\mathbf{Z} \rightarrow \mathbf{Q}$ and $\mathbf{Z} \rightarrow \mathbf{Z} / p \mathbf{Z}$ as in (13), we have

$$
\begin{array}{ll}
\operatorname{cl}_{\mathbf{Z}}(\{0\})=\{0\}, & \operatorname{cl}_{\mathbf{Z}}^{\prime}(\{0\})=p \mathbf{Z} \\
\operatorname{cl}_{\mathbf{Z}}(p \mathbf{Z})=\mathbf{Z}, & \operatorname{cl}_{\mathbf{Z}}^{\prime}(p \mathbf{Z})=p \mathbf{Z},
\end{array}
$$


so neither closure operator majorizes the other.

Nevertheless, one has the feeling that $\mathbf{Z} / p \mathbf{Z}$ satisfies "more rational relations" than $\mathbf{Q}$. This idea of comparing sets of rational relations can in fact be formalized, and related to the following concept.

Definition 32. If $f: R \rightarrow D, f^{\prime}: R \rightarrow D^{\prime}$ are homomorphisms of a ring into two division rings, then a specialization s from $D$ to $D^{\prime}$ over $R$ means a homomorphism from a subring $D_{s}$ of $D$, containing the image of $R$, to $D^{\prime}$, which makes a commuting triangle with the maps from $R$, and which has the property that

$$
\left(\forall a \in D_{s}\right) \quad s(a) \neq 0 \Rightarrow a^{-1} \in D_{s},
$$

equivalently, that $D_{s}$ is a local subring of $D$ and $\operatorname{Ker}(s)$ is its maximal ideal.

For instance, we see that over $R=\mathbf{Z}$ there is a specialization in this sense from $\mathbf{Q}$ to $\mathbf{Z} / p \mathbf{Z}$, whose domain is the local $\operatorname{ring} \mathbf{Z}_{(p)}$ of rational numbers with denominator prime to $p$. It is shown in [1, $\S \S 1-2]$ that if one formalizes appropriately the concept of rational relation, then a specialization from $D$ to $D^{\prime}$ over $R$ exists if and only if $D^{\prime}$ satisfies all rational relations in the elements of $R$ that hold in $D$.

We shall not develop here the theory of rational relations; but below, we shall determine the condition that must be satisfied by two coherent matroidal structures on free $R$-modules for there to exist a specialization over $R$ between the corresponding division rings.

Definition 33. If $\mathrm{cl}$ is a closure operator on a set $F$, let us call a finite family of elements $x_{1}, \ldots, x_{n} \in F$ dependent with respect to $\mathrm{cl}$ if for some $i, x_{i} \in$ $\operatorname{cl}\left(\left\{x_{1}, \ldots, x_{i-1}, x_{i+1}, \ldots, x_{n}\right\}\right)$; otherwise we will call $x_{1}, \ldots, x_{n}$ independent. We shall likewise call a set (as distinct from an indexed family) of $n$ elements dependent (independent) if, when indexed in any one-to-one fashion by $1, \ldots, n$, it becomes a dependent (independent) family.

In the preceding sections, I avoided calling on even basic results from the theory of matroids, but used only direct arguments from the concept of closure operator together with the exchange axiom; but in the discussion below, I will take for granted some basic facts; in particular, that in a matroid $F$ which is the closure of a finite set of elements, if $n$ is the least cardinality of such a set, then sets of $n$ elements having $F$ as closure, minimal subsets having $F$ as closure, independent subsets (Definition 33) having $F$ as closure, independent sets of $n$ elements, and maximal independent subsets, all coincide. Such sets are usually called bases of $F$, and $n$ the rank of $F$, but since we will also be dealing with module-theoretic bases and the module-theoretic rank of a free module, we will introduce a distinguishing prefix for the matroidal concepts. As the lemma that follows shows, however, there are relations between the two kinds of bases and rank.

Definition 34. In the situation of the preceding paragraph, a set with the indicated equivalent properties will be called a cl-basis of $F$, and the integer $n$ will be called the cl-rank of $F$.

Lemma 35. If $\left(\mathrm{cl}_{R^{n}}\right)_{n \in \mathbf{N}}$ is a proper coherent matroidal structure on free $R$-modules, then for each $n$, any module-theoretic basis $\left\{b_{1}, \ldots, b_{n}\right\}$ of $R^{n}$ is also a cl-basis.

Proof. By (8) $\operatorname{cl}_{R^{n}}\left(\left\{b_{1}, \ldots, b_{n}\right\}\right)=R^{n}$, so we need only prove that $b_{1}, \ldots, b_{n}$ are independent, i.e., that no $b_{i}$ is in the cl-closure of the set formed by the others. 
From Lemma [16 we know that $R / \operatorname{cl}_{R}(\{0\})$ is a nonzero cl-simple (hence clsemisimple) $R$-module. Hence for each $i$, the kernel of the homomorphism of $R^{n}$ onto that module taking $b_{i}$ to [1] and all other $b_{j}$ to 0 will be a cl-closed submodule. This submodule contains the other $b_{j}$ but not $b_{i}$, proving the desired independence.

We are now ready to look at order relations between coherent matroidal structures. The following definition includes both the relation which we noted did not hold between the coherent matroidal structures of (13), and the relation which we will soon see corresponds to the existence of a specialization, and so does hold in that case.

Definition 36. If $\mathrm{cl}$ and $\mathrm{cl}^{\prime}$ are two finitary closure operators on the same set $F$, we shall write

$$
\mathrm{cl} \leq \mathrm{cl}^{\prime}
$$

if for all $X \subseteq F$ we have $\mathrm{cl}(X) \subseteq \operatorname{cl}^{\prime}(X)$, while we will write

$$
\mathrm{cl} \preccurlyeq \mathrm{cl}^{\prime}
$$

if every finite family of elements of $F$ which is dependent with respect to cl is also dependent with respect to $\mathrm{cl}^{\prime}$; equivalently, if every family independent with respect to $\mathrm{cl}^{\prime}$ is independent with respect to $\mathrm{cl}$.

If $R$ is a ring and $\left(\mathrm{cl}_{R^{n}}\right)_{n \in \mathbf{N}},\left(\mathrm{cl}_{R^{n}}^{\prime}\right)_{n \in \mathbf{N}}$ are families of finitary closure operators on free $R$-modules of finite rank, we shall frequently abbreviate the names of these families to $\mathrm{cl}$ and $\mathrm{cl}^{\prime}$, and write $\mathrm{cl} \leq \mathrm{cl}^{\prime}$ as an abbreviation for the statement that for all $n, \mathrm{cl}_{R^{n}} \leq \mathrm{cl}_{R^{n}}^{\prime}$, and $\mathrm{cl} \preccurlyeq \mathrm{cl}^{\prime}$ as an abbreviation for the statement that for all $n, \mathrm{cl}_{R^{n}} \preccurlyeq \mathrm{cl}_{R^{n}}^{\prime}$.

Clearly, $\leq$ is a partial ordering on the closure operators on a set, and $\preccurlyeq$ is a preordering. In general, $\preccurlyeq$ is not a partial ordering. For instance, if we let $\mathrm{cl}$ be the closure operator on $\{0,1\}$ having closed sets $\varnothing,\{0\}$ and $\{0,1\}$, and $\mathrm{cl}^{\prime}$ the operator having closed sets $\varnothing$ and $\{0,1\}$, then for both operators the only dependent subset is the whole set, so they are equivalent under $\preccurlyeq$. On the other hand, a matroid structure is known to be determined by its set of dependent families [15 §1.2], so distinct matroid structures cannot be $\preccurlyeq$-equivalent; hence when restricted to the set of matroid structures, $\preccurlyeq$ is a partial order.

Lemma 37. Suppose $\mathrm{cl}, \mathrm{cl}^{\prime}$ are closure operators on a set $F$. Then

$$
\mathrm{cl} \leq \mathrm{cl}^{\prime} \Rightarrow\left(\begin{array}{c}
\text { for every } n \text { and every } \mathrm{cl}^{\prime} \text {-independent family } \\
x_{1}, \ldots, x_{n} \text {, one has } \operatorname{cl}\left(\left\{x_{1}, \ldots, x_{n}\right\}\right) \subseteq \operatorname{cl}^{\prime}\left(\left\{x_{1}, \ldots, x_{n}\right\}\right)
\end{array}\right) \Rightarrow \mathrm{cl} \preccurlyeq \mathrm{cl}^{\prime} \text {. }
$$

Moreover, if $\mathrm{cl}^{\prime}$ is a matroid structure, the last implication is reversible. Thus, on matroid structures, the relation $\preccurlyeq$ is equivalent to the middle condition.

Proof. The first implication is trivial. To see the second, assume the middle condition and let $x_{1}, \ldots, x_{n}$ be a $\mathrm{cl}^{\prime}$-independent family. If it were cl-dependent, i.e., if for some $i, x_{i} \in \operatorname{cl}\left(\left\{x_{1}, \ldots, x_{i-1}, x_{i+1}, \ldots, x_{n}\right\}\right)$, this would be a contradiction to the middle condition applied to the $\mathrm{cl}^{\prime}$-independent family $x_{1}, \ldots, x_{i-1}, x_{i+1}, \ldots, x_{n}$.

Finally, assume $\mathrm{cl}^{\prime}$ is a matroid structure, $\mathrm{cl} \preccurlyeq \mathrm{cl}^{\prime}$, and $x_{1}, \ldots, x_{n}$ is a $\mathrm{cl}^{\prime}$ independent family. If $x_{n+1}$ is any element of $\operatorname{cl}\left(\left\{x_{1}, \ldots, x_{n}\right\}\right)$, then the family $x_{1}, \ldots, x_{n+1}$ is cl-dependent, hence as $\mathrm{cl} \preccurlyeq \mathrm{cl}^{\prime}$, it is $\mathrm{cl}^{\prime}$-dependent. Thus we have some relation $x_{i} \in \operatorname{cl}^{\prime}\left(\left\{x_{1}, \ldots, x_{i-1}, x_{i+1}, \ldots, x_{n+1}\right\}\right)(i \leq n+1)$. If $i \neq n+1$, the 
exchange axiom (15) and the $\mathrm{cl}^{\prime}$-independence of $x_{1}, \ldots, x_{n}$ allow us to get from this relation the condition $x_{n+1} \in \operatorname{cl}^{\prime}\left(\left\{x_{1}, \ldots, x_{n}\right\}\right)$, while if $i=n+1$, that is given. This proves the middle condition of the display.

Comparing the leftmost and the middle conditions of the above lemma, we see that the distinction between the relations $\leq$ and $\preccurlyeq$ on matroid structures is a subtle one. In view of the apparent unnaturalness of the relation $\preccurlyeq$ compared with $\leq$, the reader should be properly skeptical, and verify that for the matroidal structures of (13), even though, as noted at the beginning of this section, $\mathrm{cl} \leq \mathrm{cl}^{\prime}$ fails, $\mathrm{cl} \preccurlyeq \mathrm{cl}^{\prime}$ holds.

We remark that one can have two proper matroid structures on a set that are comparable under $\leq$. For instance, if $k \subseteq K$ are fields, then on any $K$-vectorspace, the matroid structures arising from the $k$ - and $K$-vector-space structures are $\leq$-comparable. However, the matroid structure arising from the $k$-vector-space structure fails to satisfy (6) for $R=K$; so this is not an example of two $\leq$ comparable coherent matroidal structures on free $K$-modules. The interested reader can in fact prove that no two proper coherent matroidal structures on free modules over a ring $R$ can be comparable under $\leq$, using the results of the preceding section, in particular, Lemma 27] But I have defined $\leq$ here only to provide perspective on the less obvious relation $\preccurlyeq$, which we shall now study. Thus,

In this section unless the contrary is stated, $R$ will be a ring, and $\left(\mathrm{cl}_{R^{n}}\right)_{n \in \mathbf{N}},\left(\mathrm{cl}_{R^{n}}^{\prime}\right)_{n \in \mathbf{N}}$ will be two proper coherent matroidal structures on free $R$-modules, satisfying $\mathrm{cl} \preccurlyeq \mathrm{cl}^{\prime}$.

Caveat: This does not imply that for all finitely generated $R$-modules $M, \mathrm{cl}_{M} \preccurlyeq$ $\mathrm{cl}_{M}^{\prime}$. For instance, again considering the matroidal structures of (13), which satisfy $\mathrm{cl} \preccurlyeq \mathrm{cl}^{\prime}$, we find that in $M=\mathbf{Z} / p \mathbf{Z}$, the 1-element family ([1]) is cl-dependent but $\mathrm{cl}^{\prime}$-independent; so $\mathrm{cl}_{M} \npreceq \mathrm{cl}_{M}^{\prime}$. Hence below, in studying the relation between $\mathrm{cl}_{M}$ and $\mathrm{cl}_{M}^{\prime}$ for a general module $M$, our method will be to write $M$ as an image of a free module $R^{n}$, and apply the definition of $\preccurlyeq$ there. Our key concept will be that of

Definition 38. By a $\mathrm{cl}^{\prime}$-stable pointed cl-simple $R$-module, we shall mean a pointed cl-simple $R$-module $(M, p)$ such that, writing $\bar{p}$ for the image of $p$ in $M / \mathrm{cl}_{M}^{\prime}(\{0\})$, the pair $\left(M / \mathrm{cl}_{M}^{\prime}(\{0\}), \bar{p}\right)$ is a pointed $\mathrm{cl}^{\prime}$-simple $R$-module.

In that situation, we shall denote the latter pointed module $(M, p)^{\prime}$.

Let us show that these objects are plentiful. Suppose we take any pointed $\mathrm{cl}^{\prime}-$ simple $R$-module $\left(M^{\prime}, p^{\prime}\right)$, and write $M^{\prime}$ as $R^{n} / N^{\prime}$ for some $\mathrm{cl}^{\prime}$-closed submodule $N^{\prime} \subseteq R^{n}$. Then taking a $\mathrm{cl}^{\prime}$-independent subset of $R^{n}$ having $\mathrm{cl}^{\prime}$-closure $N^{\prime}$, and adjoining to it an inverse image of $p^{\prime}$, we get a cl'-basis $x_{1}, \ldots, x_{n}$ of $R^{n}$ such that $N^{\prime}=\operatorname{cl}_{R^{n}}^{\prime}\left(\left\{x_{1}, \ldots, x_{n-1}\right\}\right)$, and $p^{\prime} \in M^{\prime}$ is the image of $x_{n}$. In view of the relation $\mathrm{cl} \preccurlyeq \mathrm{cl}^{\prime}$, the $n$-tuple $x_{1}, \ldots, x_{n}$ is also cl-independent, hence as $R^{n}$ has the same rank, $n$, with respect to $\mathrm{cl}$ as with respect to $\mathrm{cl}^{\prime}$ (by Lemma 35), this family will also be a cl-basis of $R^{n}$. If we now let $N=\operatorname{cl}_{R^{n}}\left(\left\{x_{1}, \ldots, x_{n-1}\right\}\right)$, the final paragraph of Lemma 37 shows that $N \subseteq N^{\prime}$, whence $\mathrm{cl}_{R^{n}}^{\prime}(N)=N^{\prime}$. Letting $M=R^{n} / N$ and letting $p$ be the image of $x_{n}$ in that module, we find that $(M, p)$ is a $\mathrm{cl}^{\prime}$-stable pointed cl-simple module with $(M, p)^{\prime}=\left(M^{\prime}, p^{\prime}\right)$. Thus, every pointed $\mathrm{cl}^{\prime}$-simple module $\left(M^{\prime}, p^{\prime}\right)$ arises as $(M, p)^{\prime}$ for some $\mathrm{cl}^{\prime}$-stable pointed cl-simple module $(M, p)$. 
Now by (7) applied to $\mathrm{cl}^{\prime}$, every homomorphism $f: M_{1} \rightarrow M_{2}$ of finitely generated $R$-modules induces a homomorphism $f^{\prime}$ of $\mathrm{cl}^{\prime}$-semisimple $R$-modules making a commutative diagram,

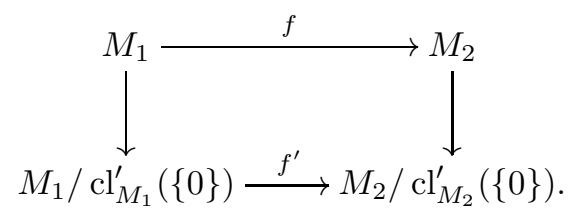

In particular, any map $\left(M_{1}, p_{1}\right) \rightarrow\left(M_{2}, p_{2}\right)$ of $\mathrm{cl}^{\prime}$-stable pointed cl-simple $R$ modules induces a map $\left(M_{1}, p_{1}\right)^{\prime} \rightarrow\left(M_{2}, p_{2}\right)^{\prime}$ of pointed $\mathrm{cl}^{\prime}$-simple $R$-modules.

In Lemma 17 we described the least upper bound $\left(M_{3}, p_{3}\right)$ of two pointed clsimple modules $\left(M_{1}, p_{1}\right)$ and $\left(M_{2}, p_{2}\right)$. Suppose now that $\left(M_{1}, p_{1}\right)$ and $\left(M_{2}, p_{2}\right)$ are $\mathrm{cl}^{\prime}$-stable. It is not hard to see from its explicit description in Lemma 17 that the least upper bound construction "commutes" with the $(M, p)^{\prime}$ construction, in the sense that $\left(M_{3}, p_{3}\right)$ will also be $\mathrm{cl}^{\prime}$-stable, and $\left(M_{3}, p_{3}\right)^{\prime}$ will be the least upper bound of $\left(M_{1}, p_{1}\right)^{\prime}$ and $\left(M_{2}, p_{2}\right)^{\prime}$ in the partially ordered set of pointed cl'-simple modules. (Key step in verification: if $\left(M_{1}, p_{1}\right)$ is expressed as above in terms of a free module $R^{m}$ and a cl'-basis $x_{1}, \ldots, x_{m}$, and $\left(M_{2}, p_{2}\right)$ similarly in terms of $R^{n}$ and $y_{1}, \ldots, y_{n}$, then $\left(M_{3}, p_{3}\right)$ may be expressed using $R^{m+n}$ and the cl'-basis $x_{1}, \ldots, x_{m-1}, y_{1}, \ldots, y_{n-1}, x_{m}-y_{n}, x_{m}$.) Hence $\mathrm{cl}^{\prime}$-stable pointed cl-simple modules form a directed subsystem of the system of all pointed cl-simple modules, and the canonical morphisms $M \rightarrow M / \mathrm{cl}_{M}^{\prime}(\{0\})$ map this system onto the directed system of all pointed $\mathrm{cl}^{\prime}$-simple modules.

Since all maps in the directed system of pointed cl-simple modules are one-to-one, so that objects of that system embed in the direct limit object $D_{R}$, the direct limit of our subsystem of $\mathrm{cl}^{\prime}$-stable objects can be identified with a submodule of $D_{R}$; let us call this $\left(D_{s}\right)_{R} \subseteq D_{R}$. If we denote by $D_{R}^{\prime}$ the direct limit of all pointed cl'-simple modules, our map between directed systems gives us an $R$-module homomorphism

$$
s_{R}:\left(D_{s}\right)_{R} \rightarrow D_{R}^{\prime}
$$

Let $\mathbf{m}$ denote the kernel of (29). We note:

Let $x \in\left(D_{s}\right)_{R}$ be the image in the direct-limit module $\left(D_{s}\right)_{R}$ of an element $q$ of a $\mathrm{cl}^{\prime}$-stable pointed cl-simple module $(M, p)$. Then $x \in \mathbf{m}$ if and only if $q \in \mathrm{cl}_{M}^{\prime}(\{0\})$.

From this we can deduce

Lemma 39. Let $a$ be any endomorphism of the $R$-module $\left(D_{s}\right)_{R}$. Then

(i) $a(\mathbf{m}) \subseteq \mathbf{m}$.

(ii) There exists a unique endomorphism $a^{\prime}$ of $D_{R}^{\prime}$ such that $s_{R} a=a^{\prime} s_{R}$.

(iii) Either a has range in $\mathbf{m}$, or it carries $\left(D_{s}\right)_{R}-\mathbf{m}$ into $\left(D_{s}\right)_{R}-\mathbf{m}$.

Proof. The idea of (i) is to apply (7) to the closure shown in (30). In detail: Given $x \in \mathbf{m}$, take $\left(M_{1}, p_{1}\right)$ and $q \in M_{1}$ such that the unique basepoint-respecting map $h_{1}: M_{1} \rightarrow\left(D_{s}\right)_{R}$ carries $q$ to $x$. Now $a\left(h_{1}\left(M_{1}\right)\right)$ is a finitely generated submodule of $\left(D_{s}\right)_{R}$, hence lies in the image $h_{2}\left(M_{2}\right)$ of some cl'-stable pointed clsimple $R$-module $\left(M_{2}, p_{2}\right)$; so as $h_{2}$ is one-to-one, we have a module homomorphism $b: M_{1} \rightarrow M_{2}$ such that $a h_{1}=h_{2} b$. By (30), $q \in \mathrm{cl}_{M_{1}}^{\prime}(\{0\})$. Applying (77), we get $b(q) \in \mathrm{cl}_{M_{2}}^{\prime}(\{0\})$, so $h_{2} b(q) \in \mathbf{m}$; but $h_{2} b(q)=a h_{1}(q)=a(x)$, so $a(x) \in \mathbf{m}$, as required. 
Since $s_{R}$ is surjective with kernel $\mathbf{m}$, (i) implies (ii). Combining this with the fact that every endomorphism of $D_{R}^{\prime}$ is either zero or one-to-one (Lemma 19]), we get (iii).

The above result relates $\left(D_{s}\right)_{R}$ and $D_{R}^{\prime}$; let us now relate $\left(D_{s}\right)_{R}$ with $D_{R}$. Mimicking the proof of Lemma 19, we find that for every $d \in\left(D_{s}\right)_{R}-\mathbf{m}$, there will be an endomorphism of $\left(D_{s}\right)_{R}$ taking $d$ to 1 . Now by Lemma 23, every endomorphism of $\left(D_{s}\right)_{R}$ is the restriction of a unique endomorphism of $D_{R}$, so the above result means that $\left(D_{s}\right)_{R}$ is closed under left multiplication in $D$ by $d^{-1}$. Since the image of $\left(D_{s}\right)_{R}$ under that left multiplication map contains $1 \notin \mathbf{m}$, point (iii) of the above lemma tells us that that map takes $\left(D_{s}\right)_{R}-\mathbf{m}$ into itself; so in particular, the image of 1 under that map, $d^{-1}$, is in $\left(D_{s}\right)_{R}-\mathbf{m}$. Hence we can apply the same result with $d^{-1}$ in the role of $d$, and conclude that $\left(D_{s}\right)_{R}$ is closed under left multiplication by $\left(d^{-1}\right)^{-1}=d$.

Thus the set of elements $d \in\left(D_{s}\right)_{R}$ such that $\left(D_{s}\right)_{R}$ is closed under left multiplication by $d$ contains $\left(D_{s}\right)_{R}-\mathbf{m}$. But that set is closed under addition, hence is all of $\left(D_{s}\right)_{R}$; i.e., $\left(D_{s}\right)_{R}$ is the underlying $R$-module of a subring $D_{s} \subseteq D$. Since $1 R \subseteq\left(D_{s}\right)_{R}$, this subring contains $f(R)$.

From the definition of the ring structures of $D$ and $D^{\prime}$, we see that the module homomorphism $s_{R}$ is a ring homomorphism, which we will write $s: D_{s} \rightarrow D^{\prime}$. We have seen that its kernel, $\mathbf{m}$, has the property that $D_{s}$ contains the inverses of all elements of $D_{s}-\mathbf{m}$; hence $D_{s}$ is a local ring, $\mathbf{m}$ its maximal ideal, and $D^{\prime}$ its residue ring. Since $s$ clearly makes a commuting triangle with our maps of $R$ into $D_{s}$ and $D^{\prime}$, we have proved

Proposition 40. Let $R$ be a ring, let $\mathrm{cl}$, $\mathrm{cl}^{\prime}$ be coherent matroidal structures on free $R$-modules, and let $D, D^{\prime}$ be the division rings constructed from these. Then if $\mathrm{cl} \preccurlyeq \mathrm{cl}^{\prime}$, there exists a specialization s from $D$ to $D^{\prime}$ over $R$.

The converse is easier to prove. I have left it to the end because the shortest proof from standard results involves matrix arguments, and I wanted to do as much as possible without these.

Proposition 41. Let $f: R \rightarrow D$ and $f^{\prime}: R \rightarrow D^{\prime}$ be homomorphisms from a ring $R$ into two division rings, and $\mathrm{cl}, \mathrm{cl}^{\prime}$ the coherent matroidal structures on free $R$-modules that these induce, and suppose there exists a specialization s from $D$ to $D^{\prime}$ over $R$. Then $\mathrm{cl} \preccurlyeq \mathrm{cl}^{\prime}$.

Proof. Given $x_{1}, \ldots, x_{m} \in R^{n}$ such that (in the notation of Corollary 7$) \bar{f}^{\prime}\left(x_{1}\right), \ldots$, $\bar{f}^{\prime}\left(x_{m}\right) \in D^{\prime n}$ are linearly independent over $D^{\prime}$, we need to prove that $\bar{f}\left(x_{1}\right), \ldots$, $\bar{f}\left(x_{m}\right) \in D^{n}$ are linearly independent over $D$.

Letting $D_{s}$ denote the domain of the specialization $s$, and writing $y_{i}$ for $\bar{f}\left(x_{i}\right) \in$ $\left(D_{s}\right)^{n}$, we see that it suffices to prove that if $y_{1}, \ldots, y_{m} \in\left(D_{s}\right)^{n} \subseteq D^{n}$ are any elements such that $\bar{s}\left(y_{1}\right), \ldots, \bar{s}\left(y_{m}\right) \in D^{\prime n}$ are $D^{\prime}$-linearly independent, then $y_{1}, \ldots, y_{m}$ are $D$-linearly independent.

Translating this into matrix terms, we need to show that if $A$ is an $m \times n$ matrix over $D_{s}$ whose image over $D^{\prime}$ has rank $m$, then $A$ has rank $m$ as a matrix over $D$. Now from general linear algebra we know that by dropping columns from our $m \times n$ image matrix over $D^{\prime}$, we can get an $m \times m$ matrix which still has rank $m$, i.e., is invertible. Dropping the corresponding columns from $A$, we get a matrix $B$ over $D_{s}$ which is "invertible modulo the maximal ideal $\mathbf{m}$ ". But the maximal ideal 
of a local ring is its Jacobson radical so, being invertible modulo this ideal, $B$ will be invertible as a matrix over $D_{s}$. Hence it is invertible as a matrix over $D$, hence its $m$ rows are right linearly independent over $D$, hence so were the $m$ rows of the original matrix $A$, as required.

Propositions 40 and 41 together give the desired criterion for the existence of specializations.

Let us now look back at the construction we gave in the preceding section of a coherent matroidal structure $\left(\operatorname{cl}_{R^{n}}\right)_{n \in \mathbf{N}}$ on free modules over any right fir $R$. I claim that that structure is a $\preccurlyeq$-least element in the set of all coherent matroidal structures on free $R$-modules. Indeed, let $x_{1}, \ldots, x_{m}$ be any family of elements of $R^{n}$ which are cl-dependent. Then from the definition of $\mathrm{cl}$ we can see that $x_{1}, \ldots, x_{m}$ lie in a submodule of $R^{n}$ generated by fewer than $m$ elements, say $\left\{x_{1}, \ldots, x_{m}\right\} \subseteq\left\{y_{1}, \ldots, y_{r}\right\} R$ with $r<m$. Hence if $\mathrm{cl}^{\prime}$ is any other coherent matroidal structure on $R^{n}$, we have

$$
\left\{x_{1}, \ldots, x_{m}\right\} \subseteq\left\{y_{1}, \ldots, y_{r}\right\} R \subseteq \operatorname{cl}^{\prime}\left(\left\{y_{1}, \ldots, y_{r}\right\}\right) .
$$

That is, $x_{1}, \ldots, x_{m}$ lie in the $\mathrm{cl}^{\prime}$-closure of a set of fewer than $m$ elements, from which it follows (by general facts about matroids, e.g., by looking at extensions of independent subsets to bases) that $x_{1}, \ldots, x_{m}$ are $\mathrm{cl}^{\prime}$-dependent. Thus $\mathrm{cl} \preccurlyeq \mathrm{cl}^{\prime}$, as claimed.

By Proposition 40 this means that the division ring $D$ constructed from $\mathrm{cl}$ admits specializations over $R$ to all division rings with homomorphisms of $R$ into them. This is the universal property proved by Cohn, referred to near the end of the preceding section. In particular, if we take for $R$ a free associative algebra on a set $X$, the resulting universal property makes $D$ a "free division algebra on $X$ " [7] p. 244].

If $R$ is a commutative integral domain, I claim that the coherent matroidal structure cl on free $R$-modules induced by the homomorphism of $R$ into its field of fractions $K$ is, similarly, a $\preccurlyeq$-least element of the partially ordered set of all coherent matroidal structures on free $R$-modules, and hence that $K$ is similarly universal with respect to specializations. One can verify that $\mathrm{cl}$ is a $\preccurlyeq$-least element with the help of the observation that if a square matrix over $R$ is singular over $K$, it has determinant zero over $R$, hence the same is true of its images over all rings $R / P(P$ a prime ideal). Alternatively, one can prove the universal property of $K$ directly by constructing the necessary specializations using the theory of localizations of commutative rings.

On the other hand, the division ring of fractions of a noncommutative right or left Ore ring does not in general have the above universal property. To get a counterexample, let $S$ be any ring admitting embeddings in two division rings $E_{1}, E_{2}$ such that there is no specialization from $E_{1}$ to $E_{2}$ over $S$. Adjoining to $E_{1}$ a central indeterminate $t$, we get a polynomial ring $E_{1}[t]$; let $R$ be the subring of $E_{1}[t]$ consisting of elements with constant term in $S$. This will be right Ore, for given two nonzero elements $a, b \in R$, they will have a nonzero common right multiple $a u=b v$ in the Ore polynomial ring $E_{1}[t]$, and so $a u t=b v t$ will be a common right multiple of these elements in $R$. (Similarly, $R$ will be left Ore.)

Now the (right and left) division ring of fractions $D$ of $R$ clearly coincides with the division ring of fractions $E_{1}(t)$ of $E_{1}[t]$, so the division subring of $D=E_{1}(t)$ generated by the subring $S \subseteq R$ of constant polynomials is isomorphic to $E_{1}$. On 
the other hand, we get a homomorphism $R \rightarrow E_{2}$ by composing the augmentation (constant-term) map $R \rightarrow S$ with the embedding $S \rightarrow E_{2}$. If $D$ were universal with respect to specializations over $R$, it would admit a specialization to $E_{2}$ making a commuting diagram with this composite homomorphism, and this would restrict to a specialization from $E_{1}$ and $E_{2}$ over $S$, which by assumption does not exist. (This example is [5, Exercise 7.2.10, p. 395].)

It is noted in [2] $(=[7$, Chapter 7$])$ that the condition "there exists a specialization from $D$ to $D^{\prime}$ over $R$ " is just the two-division-ring case of a class of conditions that become much more varied as the number of division rings being compared increases. In [2, Theorem 12.1], [7 Theorem 7.6.10] it is noted how to translate these conditions into statements about ranks of matrices. The module-theoretic approach of the present section can probably be adapted to the study of these conditions; but I have not examined this question.

I will end this note with two appendices, which give additional perspectives on a couple of the concepts we have introduced. These depend, for the most part, only on $\S \sqrt{2}$ प above.

\section{Appendix: Another look AT Cl-Simple $R$-Modules}

The approach of $\S 92$ 4 was, roughly,

$$
\begin{aligned}
& \left(\begin{array}{c}
\text { homomorphism } \\
\text { from } R \text { to } \\
\text { division ring }
\end{array}\right) \rightarrow\left(\begin{array}{c}
\text { oper- } \\
\text { ations } \\
\mathrm{cl}_{R^{n}}
\end{array}\right) \rightarrow\left(\begin{array}{c}
\text { oper- } \\
\text { ations } \\
\mathrm{cl}_{M}
\end{array}\right) \\
& \quad \rightarrow\left(\begin{array}{c}
\text { concept of } \\
\text { cl-semisimple } \\
R \text {-module }
\end{array}\right) \rightarrow\left(\begin{array}{c}
\text { concept of } \\
\text { cl-simple } \\
R \text {-module }
\end{array}\right) \rightarrow\left(\begin{array}{c}
\text { homomorphism } \\
\text { from } R \text { to } \\
\text { division ring }
\end{array}\right) .
\end{aligned}
$$

(But not all the steps were as disjoint as this might suggest; e.g., in working with simple and semisimple modules we also referred to the operations $\mathrm{cl}_{R^{n}}$.) In $\$$, for the purpose of showing that these constructions composed cyclically to appropriate identity constructions, we briefly considered the composite construction

$$
\left(\begin{array}{c}
\text { homomorphism } \\
\text { from } R \text { to } \\
\text { division ring }
\end{array}\right) \rightarrow\left(\begin{array}{c}
\text { concept of } \\
\text { cl-semisimple } \\
R \text {-module }
\end{array}\right) \text {. }
$$

In this section we shall take a somewhat more detailed look at the composite

$$
\left(\begin{array}{c}
\text { homomorphism } \\
\text { from } R \text { to } \\
\text { division ring }
\end{array}\right) \rightarrow\left(\begin{array}{c}
\text { concept of } \\
\text { cl-simple } \\
R \text {-module }
\end{array}\right) \text {. }
$$

Suppose first that $f$ is a homomorphism from $R$ to any ring $S$ (not necessarily a division ring) and cl the closure operator on finitely generated $R$-modules that it leads to. We saw in Lemma 25 that the class of cl-semisimple $R$-modules could be nicely characterized in terms of the homomorphism $f$. But the analogous conditions do not give characterizations of the class of cl-simple $R$-modules; rather, one can show that:

For any cl-semisimple $R$-module $M$ :

$M \otimes_{R} S$ is a simple $S$-module $\Rightarrow M$ is cl-simple

$\Rightarrow M$ can be embedded in a simple $S$-module, 
but neither implication is reversible. (To see that the first implication holds, observe that every homomorphism of $M$ into an $S$-module factors through $M \otimes_{R} S$; for the second, map $M$ to a simple homomorphic image of $M \otimes_{R} S$. Examples showing that both implications are irreversible are easily obtained with $R$ any field and $S$ the $2 \times 2$ matrix ring over $R$.)

However, we shall see that things are better when $S$ is a division ring.

In this section, $R$ will be a ring, and $f: R \rightarrow D$ a homomorphism to a division ring, such that $D$ is generated as a division ring by $f(R)$.

Lemma 42. If $\mathrm{cl}$ is the coherent matroidal structure on free $R$-modules determined by $f$, then a finitely generated $R$-module $M$ is cl-simple if and only if the canonical map $M \rightarrow M \otimes_{R} D$ is one-to-one and $M \otimes_{R} D$ is one-dimensional over $D$.

In particular, a finitely generated $R$-submodule $M$ of $D$ itself is cl-simple if and only if the D-vector space $M \otimes_{R} D$ is one-dimensional; equivalently, if and only if the map $M \otimes_{R} D \rightarrow D$ induced by the inclusion $M \rightarrow D$ is an isomorphism.

Proof. If $M$ is cl-simple, then by cl-semisimplicity it embeds in $M \otimes_{R} D$, which is therefore positive-dimensional. If $M \otimes_{R} D$ had dimension $>1$, we could find two nonzero elements $x, y \in M$ whose images in this vector-space were linearly independent, hence we could find a vector-space homomorphism $M \otimes_{R} D \rightarrow D$ which annihilated $x$ but not $y$. The intersection of $M$ with the kernel of this homomorphism would be a proper nonzero cl-closed submodule, contradicting clsimplicity.

The reverse implication holds by the first half of 31. The final sentence clearly follows.

Since we have here taken $f: R \rightarrow D$ rather than cl as the given data, and have characterized what we had named cl-simple modules in terms of $-\otimes_{R} D$, we shall call them " $D$-simple" modules for the rest of the section. By the last sentence of the above lemma, we see that

Every nonzero cyclic $R$-submodule $x R \subseteq D$ is $D$-simple.

However, the sum of two $D$-simple $R$-submodules of $D$ need not be $D$-simple. For example, if $f$ is an embedding (say an inclusion) and $R$ is not right Ore, and we take two right linearly independent elements $x, y \in R$, then $x R$ and $y R$ are $D$-simple $R$-submodules of $D$, but their sum $M$ is free of rank 2 over $R$, so $M \otimes_{R} D$ is 2-dimensional over $D$.

Now by the results of 4 , the $D$-simple $R$-submodules of $D$ form a directed system with union $D$, which is equivalent to saying

(34) Every finite subset of $D$ is contained a submodule of $D$ that is $D$-simple.

Since we have just seen that the class of $D$-simple submodules of $D$ is not closed under sums, (34) is not evident from our present point of view. If we take the first sentence of Lemma 42 as our definition of $D$-simple module, can we prove (34) directly, without recourse to the construction of \&4]

We can. The key observation is

If $M_{1}$ and $M_{2}$ are $D$-simple submodules of $D$ having nonzero intersection, then $M_{1}+M_{2}$ is also $D$-simple. 
Indeed, in the above situation, $\left(M_{1}+M_{2}\right) \otimes_{R} D$ is a non-one-to-one image of the 2-dimensional $D$-vector-space $\left(M_{1} \oplus M_{2}\right) \otimes_{R} D$, and is nonzero because $M_{1}+M_{2}$ embeds in $D$, hence it is 1-dimensional.

It follows that to prove that every finite subset $\left\{x_{1}, \ldots, x_{n}\right\}$ of $D$ is contained in a $D$-simple submodule of $D$, it suffices to show that each successive pair $\left\{x_{1}, x_{2}\right\}$, $\left\{x_{2}, x_{3}\right\}, \ldots,\left\{x_{n-1}, x_{n}\right\}$ is contained in such a submodule; then (35) applied repeatedly will imply that the sum of these modules is $D$-simple. So it suffices to show that every pair $\{x, y\}$ of elements of $D$ is contained in a $D$-simple submodule of $D$. Since the case $x=0$ is trivial, let us assume the contrary, and left multiply by $x^{-1}$, thus reducing the problem to the case of pairs of the form $\{1, d\}$.

Now note that if $\left\{1, d_{1}\right\} \subseteq M_{1}$ and $\left\{1, d_{2}\right\} \supseteq M_{2}$, where $M_{1}$ and $M_{2}$ are $D$ simple, then $\left\{1, d_{1}-d_{2}\right\} \subseteq M_{1}+M_{2}$, which is $D$-simple because $M_{1}$ and $M_{2}$ have the nonzero element 1 in common. Similarly, $\left\{1, d_{1} d_{2}\right\} \subseteq M_{1}+d_{1} M_{2}$, which is $D$-simple because $M_{1}$ and $d_{1} M_{2}$ have $d_{1}$ in common; and if $d_{1} \neq 0$, then $\left\{1, d_{1}^{-1}\right\} \subseteq d_{1}^{-1} M_{1}$, which is $D$-simple because it is isomorphic to $M_{1}$. Thus, the set

$$
\{d \in D \mid\{1, d\} \text { is contained in a } D \text {-simple submodule of } D\}
$$

is a sub-division-ring of $D$. It also contains $f(R)$, since for every $r \in R,\{1, f(r)\}$ is contained in the cyclic submodule $1 R \subseteq D$. Hence (36) is all of $D$, as required to complete the argument.

The above discussion suggests that an interesting invariant of an element $d \in D$ is the smallest number of generators of a $D$-simple submodule of $D$ containing $\{1, d\}$. Invariants of this sort are studied in Cohn [5, p. 386 and $\S 7.7]$, where they are described in terms of sizes of matrices over $R$ that must be inverted in $D$ to obtain (in various ways) the element $d$, and where they are called (with one or another qualifying adjective) the "depth" of $d$.

We end this section with some tangential remarks. We saw in Lemma 42 that for $S$ a division ring, the first implication of (31) was reversible. But reversibility of the other implication is uncommon:

Lemma 43. For $f: R \rightarrow D$ as in (32), the following conditions are equivalent.

(i) Every finitely generated submodule of a $D$-simple right $R$-module is $D$-simple.

(ii) $f(R)$ is a right Ore ring.

Proof. Replacing $R$ by $f(R)$ if necessary, we may assume that $f$ is an inclusion. Note also that since $D$ is the right $R$-module direct limit of "all" $D$-simple right $R$-modules, (i) is equivalent to

(i') Every finitely generated right $R$-submodule of $D$ is $D$-simple.

Now assuming $\left(\mathrm{i}^{\prime}\right)$, we see that $D$ cannot contain any right $R$-submodule free of rank 2 , hence neither can $R$, proving (ii). Conversely, if (ii) holds, note that any nonzero cyclic right submodule $w x^{-1} R$ of $D$ contains a nonzero cyclic submodule of $R$, namely $w R$, hence by the Ore condition any two such submodules of $D$ have nonzero intersection. It follows using (35) that a sum of cl-simple submodules of $D$ is cl-simple, from which $\left(i^{\prime}\right)$ follows.

The above proof shows that if $f(R)$ is not right Ore, then $D$ contains free right $f(R)$-modules of all finite ranks. It is natural to ask whether under that hypothesis it must in fact contain isomorphic copies of all $D$-semisimple right $R$-modules. The answer is in general negative. For it is shown in [3] that there exists a division ring $D$ with a subring $R$ which is not right Ore and which generates $D$ as a division ring, such that $D$ is finitely generated as a right $R$-module. In that situation, $D$ 
will itself be a $D$-semisimple right $R$-module, hence so will $D^{2}$, but an embedding of the latter in $D$ as $R$-modules would also be an embedding as $D$-vector-spaces, which we know cannot exist.

On the other hand, if $R$ is a left Ore ring it is not hard to prove from Lemma 31 that every $D$-semisimple $R$-module $M$ is embeddable in a free $R$-module of finite rank, hence, if $R$ is not right Ore, is embeddable as an $R$-module in $D$. (It follows that the latter conclusion holds, more generally if there exists a ring $S$ with $R \subseteq S \subseteq D$ such that $S$ is left Ore but not right Ore.)

We remark that a $D$-semisimple module need not be a direct sum of $D$-simple modules. Indeed, if $R$ is a commutative integral domain and $D$ its field of fractions, the $D$-semisimple $R$-modules are the finitely generated torsion-free $R$-modules, and for $R$ not a Dedekind domain, these are not in general direct sums of submodules of rank 1. For an explicit counterexample, one can take for $R$ a polynomial ring $k[x, y, z]$ and for $M$ the submodule of $R^{2}$ generated by $(x, 0),(0, y)$ and $(z,-z)$, though the proof that this is not a direct sum of proper submodules is somewhat tedious.

\section{ApPENDIX: SUBFUnCTORS OF IDENTITY FUnCTORS}

This final section will examine the wider context to which the systems of closure operators considered in this note, and the systems of operators considered in the subject of torsion theories on $R$-modules, both belong, and raise the question of possible analogs of the concept of coherent matroidal structures for other sorts of objects than modules.

I mentioned in $\$$ that what are called torsion theories, pretorsion theories, and radicals on the category $\operatorname{Mod}_{R}$ of $R$-modules correspond to operators $T$ taking every $R$-module to a distinguished submodule, satisfying successively weaker lists of conditions. A still weaker concept is that of a preradical, meaning an arbitrary subfunctor $T$ of the identity functor on $R$-modules; i.e., an operator associating to every $R$-module $M$ a submodule $T(M)$ such that for all module homomorphisms $h: M \rightarrow M^{\prime}$, one has $h(T(M)) \subseteq T\left(M^{\prime}\right)$.

Now every such functor $T$ leads to a class of operators $\mathrm{cl}_{M}$ on submodules on $R$-modules. Namely, given a submodule $N$ of an $R$-module $M$, we define $\operatorname{cl}_{M}(N)$ to be the inverse image in $M$ of the submodule $T(M / N) \subseteq M / N$. These operators will always satisfy (1) and (2), but will not in general satisfy (3). For instance, if $R=\mathbf{Z}$, $p$ is a prime, and $T$ is the subfunctor of the identity given by $T(M)=\operatorname{Ann}_{M}(p)=$ $\{x \in M \mid p x=0\}$, then $\operatorname{cl}_{M}(N)=\{x \in M \mid p x \in N\}$, so $\operatorname{cl}_{M}\left(\operatorname{cl}_{M}(N)\right)=\{x \in$ $\left.M \mid p^{2} x \in N\right\}$, which is in general larger than $\mathrm{cl}_{M}(N)$. (Note that, consequently, in this section we must not think of "cl" as really meaning "closure" except when (3) is explicitly assumed.) It is easy to check that the systems $\left(\mathrm{cl}_{M}\right)_{M \in \operatorname{Ob}\left(\operatorname{Mod}_{R}\right)}$ of operators arising in this way from subfunctors $T$ of the identity are precisely those that satisfy (1) and (2) and the analogs of (6), (7)), and (17) (with $R^{n}$ etc. replaced by arbitrary $R$-modules in (6) and (7), the finite generation assumption deleted in (17), and subsets $X$ etc. replaced by submodules $N$ etc. in all conditions), and that such a family is determined by its restriction to operators on free $R$-modules (not necessarily of finite ranks), which can be any family of operators satisfying (1), (2), (66) and (7) (again, minus the finite-rank restrictions).

There is a symmetry that it is useful to note. To give a submodule $T(M) \subseteq M$ is equivalent to giving a factor-module $M / T(M)$, or, viewed symmetrically, a short 
exact sequence

$$
\{0\} \rightarrow T(M) \rightarrow M \rightarrow M / T(M) \rightarrow\{0\} .
$$

If a submodule $T(M)$ is associated to every module $M$ in a functorial way, we see that this exact sequence is also functorial in $M$. A consequence of this symmetry is that there are two natural "idempotence" conditions such a functor can satisfy: the submodule construction might be idempotent, meaning that

$$
(\forall M) T(T(M))=T(M),
$$

or the factor-module construction might be idempotent, which is equivalent to saying that

$$
(\forall M) T(M / T(M))=\{0\} .
$$

If we think in terms of the corresponding families of operators $\mathrm{cl}_{M}$, now viewing these symmetrically as associating to every pair, $N \subseteq M$, of a module and submodule, a module $\operatorname{cl}_{M}(N)$ with $N \subseteq \operatorname{cl}_{M}(N) \subseteq M$, then each idempotency condition can be thought of as saying that this construction "stabilizes" after one step: (37) in the sense that it again gives $\mathrm{cl}_{M}(N)$ when applied to the "lower" pair $N \subseteq \mathrm{cl}_{M}(N)$, (38) in the sense that it gives $\mathrm{cl}_{M}(N)$ when applied to the "upper" pair $\mathrm{cl}_{M}(N) \subseteq M$. (So (38) is the condition saying that the operators $\mathrm{cl}_{M}$ satisfy (3), i.e., are closure operators.)

A functor may satisfy (37) but not (38) (e.g., the functor noted above taking every $\mathbf{Z}$-module $M$ to the submodule $\operatorname{Ann}_{p}(M)$ ), or (38) but not (37) (e.g., the functor associating to a $\mathbf{Z}$-module $M$ the submodule $p M$; this corresponds to the operator $\mathrm{cl}_{M}^{\prime}$ of (13) ), or both (e.g., the functor associating to a Z-module $M$ the submodule of all its elements of $p$ th-power order), or neither (e.g., the functor associating to such an $M$ the submodule generated by all elements $p^{n} x$, where $n$ ranges over the positive integers, and $x$ over elements satisfying $p^{2 n} x=0$ ). If $T$ satisfies (37), it is natural to call $T(M)$ "the torsion submodule of $M$ ", since if we call a module $L$ satisfying $T(L)=L$ a "torsion module", then $T(M)$ is the largest submodule of $M$ with this property. Similarly, when (38) is satisfied, $M / T(M)$ is the "largest" homomorphic image of $M$ having the property $T(L)=\{0\}$, and may be called "the semisimple image of $M$ ". When both (37) and (38) hold, namely, when $T$ corresponds to a pre-torsion theory, then every $M$ is an extension of a torsion submodule $T(M)$ by a semisimple factor module $M / T(M)$.

As mentioned \$3. $T$ corresponds to a torsion theory if and only if it satisfies not only both (37) and (38), but also the strengthening of (37) saying that for every submodule $A \subseteq M, T(A)=A \cap T(M)$. This means, in particular, that for every $x \in M$ one can determine whether $x$ lies in $T(M)$ by checking whether $T(x R)=$ $x R$; in other words, by examining the module structure of $x R$, equivalently, the annihilator ideal of $x$ in $R$. Thus, the property of being in $T(M)$ is entirely "local", unrelated to how the element fits into the global structure of $M$. This contrasts strongly with the behavior of functors of the form $T(M)=\operatorname{Ker}\left(M \rightarrow M \otimes_{R} S\right)$ for ring homomorphisms $R \rightarrow S$, considered in the body of this note. We have seen that these satisfy one of the idempotence conditions, namely (38), and that functors of this sort arising from homomorphisms in which $S$ is a division ring are characterized by the further condition that the corresponding families of closure operators satisfy the exchange axiom (5). (In translating (5) into a statement about operators on 
modules, one would replace the subset $X$ by a submodule $N$, the subset $X \cup\{y\}$ by the submodule $N+y R$, etc.)

Can we define analogs of the concept of coherent matroidal structure for other sorts of entities than modules - for instance, for objects of a general variety $\mathbf{V}$ of algebras in the sense of universal algebra?

A factor-algebra of an algebra $M$ in a general variety $\mathbf{V}$ has the form $M / C$, where $C$ is a congruence on $M$; but congruences do not in general correspond to subalgebras of $M$. A consequence is that the various ways that one can characterize a preradical on a module category have three inequivalent analogs for a general variety $\mathbf{V}$ : the concept of a subfunctor of the identity functor; that of a construction associating to every pair consisting of a free object $F$ and a subobject $N$ a subobject $\operatorname{cl}_{F}(N)$ subject to (1), (2) and (7), and that of a functor associating to every object a factor-object. The last of these concepts is equivalent to that of a construction associating to every object $M$ a congruence $E(M)$ satisfying the analog of (7), and also to that of a construction $\mathrm{cl}$ associating to every pair consisting of a free object $F$ and congruence $C \subseteq F \times F$ a congruence $\operatorname{cl}_{F}(C)$ satisfying the analogs of (1), (21), (7).

For a subfunctor $T$ of the identity functor of a variety $\mathbf{V}$, there is not obvious way to formulate analogs of conditions (3) and (5)). On the other hand, these conditions do make sense for operators cl taking a free algebra $F$ and a subalgebra $N$ to an algebra $\operatorname{cl}_{F}(N)$, or taking a free algebra $F$ and a congruence $C$ to a congruence $\mathrm{cl}_{F}(C)$. So one might investigate operators of either of these sorts (perhaps on free algebras of finite rank) satisfying the analogs of (ID)-(7). It is not clear what we could do with such operators if we found them; however, we might be able to answer this better if we had more examples.

Let us look at two particular varieties of algebras where we can characterize structures of these sorts, though we shall find that neither will give us anything really new.

In the variety of groups, though congruences do not correspond to arbitrary subgroups, they correspond to normal subgroups; moreover, it is easy to see that a subfunctor of the identity must associate to every group a subgroup that is completely invariant (carried into itself by all group endomorphisms), hence normal. As a result, the three concepts noted above reduce in the case of groups to the last two mentioned, i.e., an operator $\mathrm{cl}$ associating in a functorial manner to every free group $F$ and subgroup $N$ an intermediate subgroup $\mathrm{cl}_{F}(N)$, respectively (translating congruences to normal subgroups) an operator cl associating in a functorial manner to every free group $F$ and normal subgroup $N$ an intermediate normal subgroup $\operatorname{cl}_{F}(N)$.

If we do not require our operators to satisfy (5), there are interesting classes of such constructions, including many that satisfy (33). (Example: The operator associating to every subgroup or normal subgroup $N$ of a free group $F$ the least subgroup $M$ containing $N$ and satisfying $x^{n} \in M(n>0) \Rightarrow x \in M$.) But suppose now that $\mathrm{cl}$ is an operator of either sort which satisfies both (3) and (5).

Assuming cl is proper (does not carry every pair $N \subseteq F$ to $F$ ), the free group $\langle x\rangle$ on one generator must satisfy $\operatorname{cl}_{\langle x\rangle}(\{e\}) \neq\langle x\rangle$. Consider now the two homomorphisms from the free group $\left\langle x_{1}, x_{2}\right\rangle$ on two generators onto $\langle x\rangle$ that act by taking one of the generators to $x$ and the other to $e$. The inverse images of $\operatorname{cl}_{\langle x\rangle}(\{e\})$ under these maps will be distinct nontrivial proper cl-closed normal subgroups of $\left\langle x_{1}, x_{2}\right\rangle$. Since $\left\langle x_{1}, x_{2}\right\rangle$ is generated as a group by two elements, it has cl-rank at most 2 . 
(Recall that the rank of a matroid $F$ can be characterized as the minimum cardinality of a set whose closure is $F$.) Hence by rank considerations, the intersection of the two kernels mentioned must be $\operatorname{cl}_{\left\langle x_{1}, x_{2}\right\rangle}(\{e\})$. Hence $\left\langle x_{1}, x_{2}\right\rangle / \operatorname{cl}_{\left\langle x_{1}, x_{2}\right\rangle}(\{e\})$ embeds in a direct product of two copies of $\langle x\rangle / \operatorname{cl}_{\langle x\rangle}(\{e\})$. But $\langle x\rangle / \operatorname{cl}_{\langle x\rangle}(\{e\})$ is abelian, hence so is $\left\langle x_{1}, x_{2}\right\rangle / \mathrm{cl}_{\left\langle x_{1}, x_{2}\right\rangle}(\{e\})$. From this one can deduce that for any free group $F$, any cl-closed subgroup of $F$ contains the commutator subgroup $F^{\prime}$. It follows that the operator $\mathrm{cl}$ is actually induced by an operator on abelian groups, i.e., a coherent matroidal structure on free $\mathbf{Z}$-modules, and gives no more information than such an operator.

For our other example, let $R$ be a ring, and for every $R$-module $M$, let $M_{\text {aff }}$ denote the algebra with the underlying set of $M$, and with operations $b_{r}(x, y)=x r+y(1-r)$ $(r \in R)$ and $t(x, y, z)=x-y+z$. This is essentially "an $R$-module which has lost track of its 0", since every translation map $x \mapsto x+a(a \in M)$ is an automorphism of $M_{\text {aff }}$, but once 0 is specified, the module-structure can be recovered using the above two operations. The algebras of the form $M_{\text {aff }}$, together with the empty algebra, form a variety, called the variety of "affine $R$-modules" by universal algebraists (e.g., 9]).

Now the congruences on $M_{\text {aff }}$ are the same as those of $M$, and hence correspond to the (ordinary) submodules of $M$. One can deduce that a functorial way of associating to each free affine $R$-module of finite rank a congruence so that the analogs of (3) - (5) hold is equivalent to what we have been calling a coherent matroidal structure on (ordinary) free $R$-modules. Though this example, like the preceding, gives nothing new, it does show that in situations where congruences do not correspond to subobjects, congruence-valued matroidal closure operators can be reasonable entities.

For a discussion of a concept equivalent to that of a system of operators cl on subalgebras satisfying the analogs of (11)-(7) (and based, incidentally, on a novel axiomatization of the matroid concept, though it is not so named), see [4] §VII.2]. (Notes: The display near the bottom of [4, p. 254] should read $\langle\langle X\rangle \theta\rangle=\langle X \theta\rangle$, and the assertion on p. 255, lines 5-6 that the "standard" dependence relation always satisfies (ii) should be deleted.) It would be of interest to examine variants of the "standard dependence relation" introduced there; e.g., the relation, suggested by the considerations of $\$ 7$ above, under which a family of $n$ elements of an algebra is called dependent if it is contained in a subalgebra generated by fewer than $n$ elements.

The concept of matroid, though beautifully uniting the theories of linear algebra over division rings and of transcendental field extensions, has shown an embarrassing scarcity of algebraic applications that do not reduce to one of those two examples. This makes particularly tantalizing the question of whether there exist "unexpected" coherent matroidal structures (in one sense or another) on classes of algebras other than modules.

\section{REFERENCES}

1. George M. Bergman, Rational relations and rational identities in division algebras, I, J. Algebra 43 (1976), 252-266. MR 55:56853a

2. _ Rational relations and rational identities in division algebras, II, J. Algebra 43 (1976), 267-297. MR 55:56853b

3. MR 85e: 16032

4. P. M. Cohn, Universal Algebra, 2nd ed., Reidel, Dordrecht, 1981. MR 82j:08001 
5. - Free Rings and their Relations, 2nd edition, London Mathematical Society Monographs, No. 19, 1985. MR 87e:16006. (First edition was London Mathematical Society Monograph No. 2, 1971; MR 51:8155. Third edition is in preparation.)

6. —_ Algebra, 2nd ed. v. 3, Wiley \& Sons, 1991. MR 92c:00001 (1st ed. MR 58:26625).

7. Skew Fields. Theory of general division rings, Encyclopedia of Mathematics and its Applications, v. 57, Cambridge Univ. Press. MR 97d:12003

8. J. L. Fisher, Embedding free algebras in skew fields, Proc. Amer. Math. Soc. 30 (1971), 453458. MR 43:7465

9. Keith A. Kearnes, Idempotent simple algebras, Logic and Algebra (Pontignano, 1994), Lecture Notes in Pure and Appl. Math., v. 180, Dekker, 1996, pp. 529-572. MR 97k:08004

10. Joachim Lambek, Torsion Theories, Additive Semantics, and Rings of Quotients, Springer Lecture Notes in Mathematics, v. 177, 1971. MR 44:1685

11. Peter Malcolmson, A prime matrix ideal yields a skew field, J. London Math. Soc. (2) 18 (1978), 221-233. MR 80d:16003

12. _ Determining homomorphisms to skew fields, J. Algebra 64 (1980), 399-413. MR 81k:16005

13. A. H. Schofield, Representation of Rings over Skew Fields, London Mathematical Society Lecture Note Series, No. 92, 1985. MR 87c:16001

14. B. L. van der Waerden, Moderne Algebra, Bd. I, Springer-Verlag, 1930. Jahrbuch 56, p. 138. (There have been several later editions, and English translations, e.g., Modern Algebra, v. I, transl. Fred Blum, Frederick Ungar Publishing Co., 1949, MR 10:587b Starting with the 1955 4th edition, the title was shortened to Algebra.)

15. D. J. A. Welsh, Matroid Theory, London Mathematical Society Monographs, No. 8, 1976. MR 55:148

16. H. Whitney, On the abstract properties of linear dependence, Amer. J. Math. 57 (1935), 509-533.

Department of Mathematics, University of California, Berkeley, California 947203840

E-mail address: gbergman@math.berkeley.edu 